\title{
The WIRCam Deep Survey
}

\section{Mass selected clustering ${ }^{\star} \star \star$}

\author{
R. M. Bielby ${ }^{1}$, V. Gonzalez-Perez ${ }^{1,2}$, H. J. McCracken ${ }^{3}$, O. Ilbert ${ }^{4}$, E. Daddi ${ }^{5}$, O. Le Fèvre ${ }^{4}$, P. Hudelot ${ }^{3}$, J.-P. Kneib ${ }^{4}$, \\ Y. Mellier ${ }^{3}$, and C. Willott ${ }^{6}$
}

1 Dept. of Physics, Durham University, South Road, Durham, DH1 3LE, UK

e-mail: rmbielby@gmail.com

2 Centre de Physique des Particules de Marseille, Aix-Marseille Université, CNRS/IN2P3, Marseille, France

3 Institut d'Astrophysique de Paris, UMR7095 CNRS, Université Pierre et Marie Curie, 98bis Boulevard Arago, 75014 Paris, France

4 Aix Marseille Université, CNRS, LAM - Laboratoire d'Astrophysique de Marseille, 38 rue F. Joliot-Curie, 13388 Marseille, France

5 Service d'Astrophysique, CEA/Saclay, 91191 Gif-sur-Yvette, France

${ }^{6}$ Herzberg Institute of Astrophysics, National Research Council, 5071 West Saanich Road, Victoria, BC V9E 2E7, Canada

Received 8 October 2013 / Accepted 9 June 2014

\section{ABSTRACT}

\begin{abstract}
We present an analysis of the clustering of galaxies from $z \approx 2$ to the present day using the WIRCam Deep Survey (WIRDS). WIRDS combines deep optical data from the CFHTLS Deep fields with its own deep near-infrared data, providing a photometric data-set over an effective area of $2.4 \mathrm{deg}^{2}$, from which accurate photometric redshifts and stellar masses can be estimated. We use the data to calculate the angular correlation function for galaxy samples split by star-formation activity, stellar mass and redshift. Using WIRDS with its large total area and multiple fields gives a low cosmic variance contribution to the error, which we estimate to be less than $\sim 2.8 \%$. Based on power-law fits, we estimate the real-space clustering for each sample, determining clustering lengths and power-law slopes. For galaxies selected by constant mass, we find that the clustering scale shows no evolution up to $z \approx 2$. Splitting the galaxy sample by mass, we see a consistent trend for higher mass galaxies to have larger clustering scales at all redshifts considered. We use our results to test the GALFORM semi-analytical model of galaxy formation and evolution. The observed trends are well matched by the model galaxies for both the redshift evolution and the mass dependence of the galaxy clustering. We split the galaxy population into passive and star-forming populations based on rest-frame dust-corrected NUV-r colours. We find that the passive galaxy populations show a significantly larger clustering scale at all redshifts than the star-forming population below masses of $M_{\star} \sim 10^{11} h^{-1} M_{\odot}$, showing that even at $z \approx 2$ passive galaxies exist in denser environments than the bulk of the star-forming galaxy population. For star-forming galaxies with stellar masses of $M_{\star} \gtrsim 10^{11} h^{-1} M_{\odot}$, we find a clustering strength of $\sim 8 h^{-1} \mathrm{Mpc}$ across all redshifts, comparable to the measurements for the passive population. Additionally, for star-forming galaxies we see that clustering strength increases for higher stellar mass systems, however little sign of a mass dependence in passive galaxies is observed over the range in stellar mass that is probed. Comparing our results to the model galaxy population produced by GALFORM, we find a qualitative good agreement between the model predictions and the observed clustering. Finally, we investigate the connection between galaxy stellar mass and dark matter halo mass, showing a clear correlation between the two in both the WIRDS data and the GALFORM predictions.
\end{abstract}

Key words. galaxies: evolution - large-scale structure of Universe - galaxies: high-redshift

\section{Introduction}

The current consensus suggests that star formation in the Universe reached its peak within the redshift range $1<z<2$, whilst $\sim 50 \%-70 \%$ of mass assembly took place in the redshift range $1<z<3$ (Connolly et al. 1997; Dickinson et al. 2003; Arnouts et al. 2007; Pozzetti et al. 2007; Noeske et al. 2007). It is evident that there is a substantial population of massive galaxies at $z>1$ and this has presented significant complications for hierarchical structure formation models. Additional observations of galaxies at $z>1$ are of paramount importance to advance our understanding of galaxy formation and evolution as a whole.

At redshifts of $z>1$, identifiable spectral features begin to move out of the optical wavelength range and near-infrared

* Based on data obtained with the European Southern Observatory Very Large Telescope, Paranal, Chile, under Large Programs 070.A-9007, 175.A-0839, and 177.A-0837.

$\star \star$ Appendices are available in electronic form at http://www . aanda.org
(NIR) observations become essential. The role of environment and large-scale structure at these redshifts is largely unexplored (Renzini \& Daddi 2009). In addition to making it possible to select galaxies in this important redshift range, NIR galaxy samples offer several advantages compared to purely optical selections (see for example Cowie et al. 1994). As $k$-corrections in $K_{\mathrm{s}}$ band are insensitive to galaxy type over a wide redshift range, NIR-selected samples provide a fairly unbiased census of galaxy populations at high redshifts (providing that the extinction is not too high, as in the case of some submillimeter galaxies). Such samples represent the ideal input catalogues from which to extract targets for spectroscopic surveys as well as for determining accurate photometric redshifts.

An important application and aim for achieving deeper NIR data is to trace galaxy formation and evolution through the period of high star-formation at $1<z<2$. Key in this is understanding the evolution of the spatial clustering of galaxies to the present day. From such analysis of the spatial distribution of galaxies, one can derive the mass of the dark matter halos 
in which they reside. It is then crucial to quantify the links that may exist between the dark matter halos and the attributes of the galaxies themselves, in particular the stellar-mass, galaxy type, luminosity and star-formation rate. In addition, given an estimate of the mass of the dark matter haloes hosting a given galaxy population (and assuming a suitable model for halo evolution) one can identify the present-day descendants of these galaxies, as has been done for Lyman break galaxies (LBGs) at $z \sim 3$ (e.g. Cooke et al. 2006; Conroy et al. 2008; Bielby et al. 2011). A few studies have attempted this for passive galaxies at $z \sim 2$, but small fields of view have made these studies somewhat sensitive to the effects of cosmic variance.

Analysing the spatial distribution of galaxies and understanding the relationship between this distribution and galaxy properties is a key element in the study of galaxy formation theory. Studies of the relationship between clustering and morphology (e.g. Davis \& Geller 1976; Iovino et al. 1993; Loveday et al. 1995; Norberg et al. 2002; Coupon et al. 2012) have for a long time registered the stronger clustering tendencies of early-type over late-type galaxies. Most recently, de la Torre et al. (2011) used HST imaging of the COSMOS field to determine the morphological types of galaxies in zCOSMOS and confirmed the stronger clustering of early type galaxies over late type galaxies to $z \approx 1$. Extensive work has also been performed on the relationship between galaxy luminosity and clustering, with greater luminosity correlating with greater clustering as observed at lower redshifts (e.g. Phillipps \& Shanks 1987; Hasegawa \& Umemura 1993; Norberg et al. 2001) and extending to $z \sim 1$ (Pollo et al. 2006; Meneux et al. 2006; Coil et al. 2008).

Extending these studies to $1<z<2$ has been made possible in the last decade through the increasing availability of deep NIR imaging data as well as extensive spectroscopic redshift surveys (Le Fèvre et al. 2005a; Coil et al. 2006). Focusing on the NIR imaging, the relative difficulty in obtaining sufficiently deep data has led many studies to focus on tracer populations identified using colour selection techniques. Perhaps the most successful of these has been the $B z K$ selection (Daddi et al. 2004), which facilitates the selection of galaxies at $z \gtrsim 1.4$ and the approximate separation of these into passive $(\mathrm{p} B z K)$ and star-forming ( $\mathrm{s} B z K)$ populations. Several surveys have applied the $B z K$ selection techniques to large samples of NIR selected galaxies. In one of the widest surveys to date, Kong et al. (2006) constructed $K$-band selected samples over a $\sim 920 \operatorname{arcmin}^{2}$ field reaching $K \approx 20.8$ and attaining $K \approx 21.8$ over a $320 \operatorname{arcmin}^{2}$ sub-field. The exploration of a field of this size made it possible to measure the clustering properties of star-forming and passive galaxy samples and to establish that passive galaxies in this redshift range are substantially more strongly clustered than star-forming ones, indicating that a galaxy-type - density relation reminiscent of the local morphology-density relation must be already in place at $z>1$.4. Subsequent studies using the MUSYC (Blanc et al. 2008), UKIDSS (Hartley et al. 2008) and COSMOS (McCracken et al. 2010) surveys have confirmed these results, whilst also establishing the continuation of the luminosity dependence of galaxy clustering beyond $z>1$ (e.g. Hayashi et al. 2007).

Complimentary studies at $z>1$ have also been performed using a variety of colour selection techniques. For example, galaxies selected as extremely red objects (EROs, isolated using a cut in the $R-K$ colour) were found to be highly clustered and indicated the existence of a $z>1$ elliptical galaxy population Daddi et al. (2000); Roche et al. (2002); Brown et al. (2005). Similarly, distant red galaxies (DRGs, selected via $J-K$ colours) have been shown to be highly clustered galaxies at $1<z<3$
(Grazian et al. 2006; Foucaud et al. 2007; Quadri et al. 2008; Kim et al. 2011), whilst optically selected galaxies have also played their part (Adelberger et al. 2005).

However, given the increasing availabilty of multi-band photometry through optical and NIR wavebands, more complex selections of galaxy populations are becoming feasible and reliable via photometric template fitting. Padilla et al. (2010) used the MUSYC survey to evaluate the clustering of galaxies to $z \approx 1.5$, showing a mild dependence on sample luminosity out to this distance. Hartley et al. (2010) went further with the UKIDDS data, analysing the clustering of the passive and star-forming galaxy populations to $z \approx 3$ and confirming the stronger clustering of the passive population over the star-forming to $z \approx 1.5$, whilst reporting little dependence of clustering on $K$ band luminosity. Interestingly they find that the clustering of star-forming galaxies increases with redshift and reaches equivalent strengths to the clustering of passive galaxies at $z>2$.

It has only been relatively recently with the advent of the deep NIR imaging surveys and extensive spectroscopic surveys that the relationship of clustering to stellar mass have begun to be deeply investigated. For example, first taking the spectroscopic based work, Li et al. (2006) presented the measured mass dependence of galaxy clustering at $z<0.3$ using SDSS data, showing an increase in clustering with mass, which became more pronounced above $M^{*}$. Moving to higher redshifts, Meneux et al. (2008, 2009) measured the clustering of mass-selected samples in the VVDS-Deep and zCOSMOS Surveys respectively, finding a clustering mass dependence in their results at redshifts up to $z \approx 1.2$.

Returning to photometric data, Foucaud et al. (2010) used Palomar Observatory Wide-field Infrared Survey (POWIS) to measure the mass dependency of galaxy clustering to $z=2$, over a combined field of view of $1.16 \mathrm{deg}^{2}$ and with $K$ depths of $\approx 23.5$. Taking the full galaxy population, they found an increase in galaxy clustering with galaxy stellar mass across a range of redshifts, whilst also noting an increase in the clustering strength with redshift for samples of the same mass range. Similarly, Wake et al. (2011) measured the clustering of galaxies as a function of mass in the $0.4 \mathrm{deg}^{2}$ of the NEWFIRM (NOAO Extremely Wide-Field Infrared Imager) Medium Band Survey (NMBS). Again they point to a strong dependence of galaxy clustering on galaxy stellar mass. In terms of any stellar mass/halo mass relation, Wake et al. (2011) see little evidence of any redshift dependence in the relationship between stellar mass and halo mass over the range $1<z<2$, but see evidence for a change in the relation when comparing to results at $z<1$ from other surveys.

In this paper, we present a study of the mass, type and redshift dependence of galaxy clustering in the WIRCam Deep Survey (WIRDS). The paper is organized as follows: Sect. 2 briefly describes the WIRDS data used here. Following this, in Sect. 3 we present the clustering analysis if galaxies in the WIRDS fields as a function of type, mass and redshift over the range $0<z<2$. Section 4 provides a summary and our conclusions.

Throughout this paper, all magnitudes are given in the $\mathrm{AB}$ system unless stated otherwise. Where relevant, we assume a $\Lambda$ CDM cosmology given by $H_{0}=100 \mathrm{hm} \mathrm{s}^{-1} \mathrm{Mpc}^{-1}$, $\Omega_{\mathrm{m}}=0.25$ and $\Omega_{\Lambda}=0.75$.

\section{Data and simulations}

The work presented here is based on data from the deep fields of the Canada-France-Hawai'i Telescope Legacy Survey 
R. M. Bielby et al.: The WIRCam Deep Survey. II.

Table 1. 50\% AB magnitude completeness limits for extended sources in the CFHTLS T0006 and WIRDS imaging used in this work (as described by Bielby et al. 2012).

\begin{tabular}{lcccccccc}
\hline \hline Field & $u$ & $r$ & $g$ & $i$ & $z$ & $J$ & $H$ & $K_{\mathrm{s}}$ \\
\hline D1 & 26.54 & 26.20 & 25.63 & 25.32 & 24.82 & 24.7 & 24.7 & 24.7 \\
D2 & 26.46 & 26.17 & 25.62 & 25.30 & 24.80 & 23.4 & 24.1 & 24.0 \\
D3 & 26.45 & 26.21 & 25.63 & 25.33 & 24.77 & 24.8 & 24.8 & 24.6 \\
D4 & 26.32 & 26.17 & 25.57 & 25.26 & 24.70 & 25.1 & 24.6 & 24.6 \\
\hline
\end{tabular}

(CFHTLS). The CFHTLS Deep incorporates four $1 \times 1 \mathrm{deg}^{2}$ survey fields (designated D1 to D4) spread across a broad range in RA and declination, the four field centre co-ordinates being: D1 02:25:59, -04:29:40; D2 10:00:28, +02:12:30; D3 14:19:27, $+52: 40: 56$; and D4 22:15:31, $-17: 43: 56$. The CFHTLS produced deep optical imaging data within these fields, whilst the WIRDS survey has added deep NIR imaging data and it is the combination of these two datasets that we use here and that are described in more detail in the two sections below. In this section, we also give an overview of the GALFORM semi-analytical galaxy formation model, the predictions of which we will confront with our results.

A key benefit of the WIRDS data is the combination of 4 distinct large deep fields, which act to reduce the impact of cosmic variance. Based on the cosmic variance cookbook (GETCV) of Moster et al. (2011), we estimate the contribution to the errors on our clustering measurements to be $\lesssim 2.8 \%$.

\subsection{Optical data}

In this work, we use the CFHTLS T0006 optical data to provide 5-band optical photometry of the galaxy population. This incorporates imaging taken with the MegaCAM imager in the Canada-France-Hawai'i Telescope (CFHT) using the ugriz MegaCAM filters. The CFHTLS provides stacked images based on either a $25 \%$ or $85 \%$ cut in terms of image quality (i.e. seeing). In this case, we use catalogues based on the $85 \%$-cut stacks. The $50 \%$ completeness limits for extended sources for each band/field combination are given in Table 1. Of particular relevance when we come to identifying limiting masses for our survey, we note that the mean $50 \% i$-band completeness limit across all four fields for extended sources is $i=25.30$ (whilst the mean $80 \% i$-band limit is $i=24.35$ ). The image quality is consistently $\approx 0.7-0.8^{\prime \prime}$ across all four fields. This consistency is one of the key benefits of using the CFHTLS data, in that all the data is homogeneous in terms of seeing, depth and filter/telescope properties. A full and thorough description and characterisation is presented by Goranova et al. (2009) and we refer the reader to this document for any further information.

\subsection{Infrared data}

WIRDS is a deep infrared imaging survey of the CFHTLS Deep fields, providing infrared data to complement the CFHTLS optical data obtained with MegaCAM. The WIRDS imaging was taken with the WIRCam detector (Puget et al. 2004) on the CFHT and a detailed discussion of the observations and data reduction of the WIRDS data is provided by Bielby et al. (2012) and McCracken et al. (2010).

The data used in this paper were taken in a series of observing run from 2005-2007 and were made in co-ordination with the COSMOS consortium. Observations were conducted using three filters: $J, H$ and $K_{\mathrm{s}}$. Transmission plots of the WIRCam $J, H$ and $K_{\mathrm{s}}$ filters are available from $\mathrm{CFHT}^{1}$. The integration times for all $J, H$ and $K_{\mathrm{s}}$ band exposures was $45 \mathrm{~s}, 15 \mathrm{~s}$ and $20 \mathrm{~s}$ respectively.

The observations were carried out in queue scheduled mode at the CFHT. Image quality constraints of $0.55^{\prime \prime}<I Q<$ $0.65^{\prime \prime}$ were requested and the observations were micro-dithered using the standard WIRCam micro-dither pattern consisting of $2 \times 2$ dither patter with offsets between consecutive dithers of 0.5 pixels. Due to the WIRCam pixel scale of $0.3^{\prime \prime} /$ pixel, this micro-dithering is required in order to produce well sampled images under our seeing constraints (and to allow matching with the CFHTLS pixel-scale of $0.186^{\prime \prime} /$ pixel). A further large-scale dithering pattern was applied to the observations to avoid gaps in the coverage due to the gaps between adjacent arrays.

The limiting magnitude depths of the NIR data are given in Table 1 (Bielby et al. 2012). The photometric catalogues on which this work is based are available at the CADC archive ${ }^{2}$.

\subsection{Photometric galaxy properties, mass constraints and colour selection}

With the wavelength coverage afforded by the combination of the CFHTLS optical data and the WIRDS NIR data, it is possible to estimate photometric redshifts and stellar masses reliably over a broad redshift range. In particular, the wavelength range afforded by this collection of filters presents the possibility of the $4000 \AA$ Areak being detectable up to $z \sim 4$.

We used the Le Phare ${ }^{3}$ code (Arnouts et al. 2002; Ilbert et al. 2006) to determine photometric redshifts and galaxy properties with a $\chi^{2}$ template-fitting method. The photo- $z$ were estimated using the median of the probability distribution function (PDFz) rather than the minimum of the $\chi^{2}$ distribution. The results of the photometric redshift estimation are presented in Bielby et al. (2012), with a full comparison to spectroscopic datasets. Below we provide an overview and derived accuracies of the photometric redshifts.

We use a number of spectroscopic redshift data-sets to calibrate the photo- $z$ data in our four fields. In the D1 field, we use spectroscopic redshifts from the VVDS Deep (Le Fèvre et al. 2005b) and Ultra-Deep (Cucciati et al. 2012; Le Fèvre et al. 2013, 2014) spectroscopic samples, both observed using VIMOS on the VLT. The VVDS Deep sample is available publicly and consists of 8981 spectroscopically observed objects over an area of $0.5 \mathrm{deg}^{2}$ in the CFHTLS D1 field. It consists of a magnitude limited sample with a limit of $I \leq 24$ and samples a redshift range of $0 \leq z \leq 5$. The Ultra-Deep sample consists of $\sim 1500$ spectra over an area of $\approx 0.14 \mathrm{deg}^{2}$ and covers a magnitude range of $22.5 \leq i \leq 24.75$. Both of the VVDS spectroscopic catalogues attribute each object a flag based on the identification. These range from 1 to 4 with 1 being most unreliable and 4 being most reliable. In addition a flag 9 is given to objects identified based on a single emission line. Using the VVDS Deep data we find an outlier rate of $\eta=3.7 \%$ and $\sigma_{\Delta z /(1+z)}=0.025$, with a median magnitude of $i_{\text {median }}^{*}=22.6$, whilst using the UltraDeep data we find an outlier rate of $\eta=4.2 \%$ and $\sigma_{\Delta z /(1+z)}=0.030$,

\footnotetext{
http://www. cfht .hawaii.edu/Instruments/Filters/

wircam. html

2 http://cadcwww. dao.nrc.ca/cfht/WIRDSTQ002.html

3 http://www . cfht . hawaii . edu/ arnouts/LEPHARE/

cfht_lephare
} 
with a median magnitude of $i_{\text {median }}^{*}=23.7$. In both cases, we use only galaxies observed with the most secure identification flags (i.e. 3 and 4). The 10th and 90th percentiles of the redshift distribution for the VVDS Deep galaxies used are $z=0.31$ and $z=1.05$ respectively, whilst for the VVDS Ultra-Deep sample used, these are $z=0.33$ and $z=1.69$.

In the D2/COSMOS field we make use of the zCOSMOS 10k data (Lilly et al. 2009), which constituted the ESO Large Proposal LP175.A-0839 and provides spectroscopic redshifts based on data acquired using VIMOS on the VLT. We find 3004 objects predominantly in the magnitude range $17.5 \leq$ $i \leq 22.5$ and over a redshift range up to $z \lesssim 1.4$, which are present in our photometric catalogue. From this data (and using only objects with spectroscopic flags of 3 or 4) we estimate an outlier rate of $\eta=1.4 \%$ and $\sigma_{\Delta z /(1+z)}=0.023$, based on a sample with median magnitude of $i_{\text {median }}^{*}=21.6$. The 10th and 90th percentiles of the redshift distribution for the zCOSMOS galaxies used are $z=0.27$ and $z=0.90$ respectively.

In D3, we use the DEEP2 DR3 redshift catalogue (Davis et al. 2003, 2007), which is based on spectroscopic observations using the Deep Imaging Multi-Object Spectrograph (DEIMOS) on Keck II. The catalogue contains 47700 unique objects, of which 2977 have a "zquality" flag of $\geq 3$ (i.e. are deemed to be reliable redshifts) and are present in our photometric catalogue. This sample predominantly covers a magnitude range of $18<i<24$ with the bulk being below a redshift of $z \lesssim 1.6$. Using the DEEP2 data, we estimate an outlier rate of $\eta=3.4 \%$ and $\sigma_{\Delta z /(1+z)}=0.027$, based on a sample with median magnitude of $i_{\text {median }}^{*}=22.5$ for the WIRDS D3 photometric catalogue. The 10th and 90th percentiles of the redshift distribution for the DEEP2 galaxies used are $z=0.29$ and $z=1.18$ respectively.

In the D4 field, we use spectra obtained using the AAOmega instrument on the Anglo-Australian Telescope (AAT) as part of a program to provide optical spectroscopy of X-ray point-sources in the CFHTLS (Stalin et al. 2010). The observations provide redshifts for 1335 galaxies, all at magnitudes of $i<22.5$ (Bielby et al. 2010b). In total, 1090 of the galaxies overlap with our photometric data, most of which are at $z \lesssim 0.8$. Based on these, we find an outlier rate of $\eta=2.1 \%$ and $\sigma_{\Delta z /(1+z)}=0.021$, with a median magnitude of $i_{\text {median }}^{*}=20.0$. The 10th and 90th percentiles of the redshift distribution for the AAOmega galaxies used are $z=0.12$ and $z=0.53$ respectively.

For the purposes of the clustering analysis, we focus on measuring the clustering of galaxy populations selected using the photometric redshifts and photometrically derived masses, maximising the use of the WIRDS catalogues. We use three overall samples, the passive galaxy sample selected via rest-frame colours, the star-forming galaxy sample selected in the same way and the third incorporating the entire catalogue. The colour selection is the same as that used by Ilbert et al. (2010) in which galaxies with rest frame, dust de-reddened colours of $N U V-r \geq$ 3.5 are classed as passive and those with $N U V-r<3.5$ are classed as star-forming. Each of these are then split into redshift and mass slices.

For all three of the passive, star-forming and complete samples, we separate the population into stellar mass bins of $10^{8.6}<$ $M\left[h^{-1} M_{\odot}\right] \leq 10^{9.6}, 10^{9.6}<M\left[h^{-1} M_{\odot}\right] \leq 10^{10.6}, 10^{10.6}<$ $M\left[h^{-1} M_{\odot}\right] \leq 10^{11}$ and $M\left[h^{-1} M_{\odot}\right]>10^{11}$.

In order to estimate the mass completeness limits of our data, we follow the method outlined by Ilbert et al. (2010). We first cut the catalogue at the mean $K$-band $50 \%$ completeness limit of $K=24.5$ and then identify the minimum stellar mass at which "most" galaxies of that stellar mass are brighter than an $i$-band

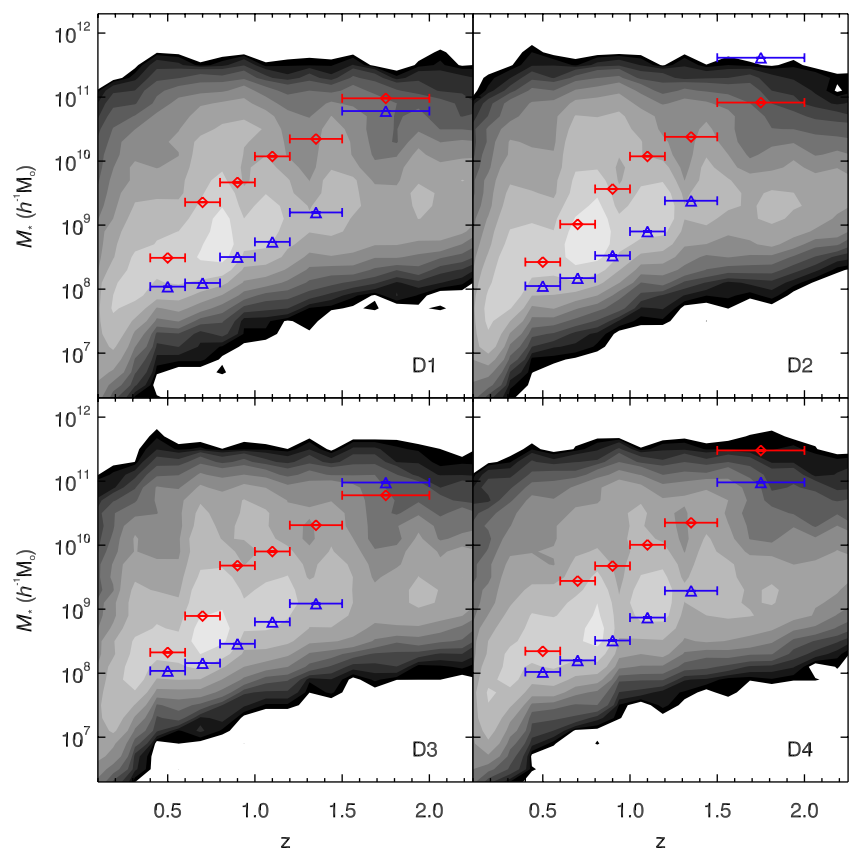

Fig. 1. Estimated mass completeness limits of the WIRDS data. The greyscale contours show the distribution of the $i<25$ galaxy population, normalized by the area of each field. Red diamonds with horizontal error bars show the estimated mass completeness limits for consecutive bins in redshift corresponding to a magnitude cut of $i=25$ for passive galaxies. Blue triangles with error bars show the same for star-forming galaxies.

limit of $i=25$ (i.e. the $\approx 70 \% i$-band completeness limit) ${ }^{4}$. As in Ilbert et al. (2010) we quantify "most" galaxies as $70 \%$ brighter than our chosen $i$-band limit. Taking the combination of the $\approx 70 \% i$-band completeness with the requirement for $70 \%$ of galaxies to be brighter than this limit, the derived minimum masses are akin to mass completeness limits of $\approx 50-60 \%$.

The estimated mass completeness limits are shown in Fig. 1 as a function of redshift and galaxy type for each of the fields. The grey-scale contour maps show the galaxy population distribution at $i<25$ normalised by the field areas. Mass completeness limits for the star-forming population are shown by the blue triangles, whilst the limits for the passive population are given by the red diamonds. The extent of each redshift-bin is given by the horizontal error bars. Estimated limits across the four fields are broadly consistent based on the imposed $i<25$ limit. In each field there is also a consistent separation between the starforming and passive galaxy mass-limits, with the star-forming galaxies probing to lower masses at $0.5 \lesssim z \lesssim 1.4$ given the $i<25$ magnitude limit.

We note that at first glance, the fact that the mass limits for the passives and star-forming galaxies become relatively consistent with each other in the $1.5<z<2$ bin, at first glance, appears to contradict what this method gives in Ilbert et al. (2010) (where the mass limit for the star-forming population is much lower than for the passive population in all their redshift bins). This is simply down to their fainter $i$-band cut of $i=25.5$ compared to our cut of $i=25$. In fact, taking their limits based on a limiting magnitude of $i=24$, gives consistent results with our $i=25$ cut, whereby the passive and star-forming populations

4 Note that an error in the text of Bielby et al. (2012) incorrectly quotes a limit of $i=25.5$ instead of the actual value of $i=25$ used in this analysis. 
R. M. Bielby et al.: The WIRCam Deep Survey. II.

Table 2. Median masses for our given $i$ and $K$ band $80 \%$ completeness limits and estimated mass completeness limits based on a maximum $30 \%$ of objects at magnitudes of $i>25$.

\begin{tabular}{|c|c|c|c|c|c|c|}
\hline \multirow[t]{3}{*}{ Redshift } & \multicolumn{3}{|c|}{ Passive } & \multicolumn{3}{|c|}{ Star-forming } \\
\hline & \multicolumn{2}{|c|}{ Median mass } & \multirow{2}{*}{$\begin{array}{c}\text { Mass limit } \\
70 \%<[i=25] \\
\left.\left.\left.M_{\odot}\right]\right)\right)\end{array}$} & \multicolumn{2}{|c|}{ Median mass } & \multirow{2}{*}{$\begin{array}{l}\text { Mass limit } \\
70 \%<[i=25] \\
\left.\left.\left.M_{\odot}\right]\right)\right)\end{array}$} \\
\hline & $i=24.4$ & $\begin{array}{c}K=24.0 \\
\left(\log _{10}\left(M\left[h^{-}\right.\right.\right.\end{array}$ & & $i=24.4$ & $\begin{array}{c}K=24.0 \\
\left(\log _{10}(M[h\right.\end{array}$ & \\
\hline $0.4<z \leq 0.6$ & 8.8 & 8.7 & 8.4 & 8.3 & 8.2 & 8.0 \\
\hline $0.6<z \leq 0.8$ & 9.3 & 8.9 & 9.4 & 8.6 & 8.5 & 8.2 \\
\hline $0.8<z \leq 1.0$ & 10.1 & 9.2 & 9.7 & 8.8 & 8.6 & 8.5 \\
\hline $1.0<z \leq 1.2$ & 10.4 & 9.3 & 10.1 & 9.2 & 8.8 & 8.9 \\
\hline $1.2<z \leq 1.5$ & 10.7 & 9.5 & 10.4 & 9.3 & 8.9 & 9.3 \\
\hline $1.5<z \leq 2.0$ & 11.0 & 9.8 & 11.0 & 9.5 & 9.2 & 11.0 \\
\hline
\end{tabular}

have the same mass limit at $1.5<z<2.0\left(M_{\star}=10^{11.3} h^{-1} M_{\odot}-\right.$ see bottom panel of their Fig. 8).

We take the median mass limit across the four fields in five redshift bins and these are given in Table 2. Given the low ratio of passive to star-forming galaxies, the limits for the entire sample are equivalent to those of the star-forming sample.

As a test of the efficacy of these derived mass limits, we calculate the median stellar mass for galaxies with magnitudes corresponding to our $80 \%$ completeness limits in $i$ and $K$. These are also given in Table 2. The median masses for the $i=24.4$ and $K=24.0$ magnitude completeness limits are all either lower or broadly consistent with the derived $70 \%$ mass completeness limits. In two cases, the median stellar mass at $i=24.4$ exceeds the $70 \%$ mass completeness limit to an extent that overlaps into the lowest accepted stellar mass bin (passive galaxies at $1.2<z \leq 1.5$ and star-forming galaxies at $0.8<z \leq 1.0$ ) and in these cases we use the median stellar mass at $i=24.4$ as the lower mass limit.

\subsection{Simulations}

We predict the clustering of mass selected galaxies in a $\Lambda \mathrm{CDM}$ universe using the GALFORM semi-analytical galaxy formation code developed by Cole et al. (2000), and extended by Benson et al. (2003), Baugh et al. (2005), Bower et al. (2006), Lagos et al. (2011) and Fanidakis et al. (2011). Semi-analytical models use physically motivated recipes and rules to follow the fate of baryons in a universe in which structures grow hierarchically through gravitational instability (see Baugh 2006, for an overview of hierarchical galaxy formation models).

In this paper we focus our attention on the Bower et al. (2006) model. Some of the key features of this model are (i) a time scale for quiescent star formation that varies with the dynamical time of the disk and which therefore changes significantly with redshift; (ii) bursts of star formation occur due to both galaxy mergers and when disks become dynamically unstable; and (iii) the inclusion of both supernova and active galactic nuclei (AGN) feedback. This feedback is implemented in such a way that AGNs are able to heat the cooling flows in massive haloes, preventing any further star formation in galaxies within such haloes. Bower et al. adopt the cosmological parameters of the Millennium Simulation (Springel et al. 2005), which are in broad agreement with constraints from measurements of the cosmic microwave background radiation and large scale galaxy clustering (e.g. Sánchez et al. 2009): $\Omega_{\mathrm{m} 0}=0.25, \Omega_{\Lambda 0}=0.75$, $\Omega_{b 0}=0.045, \sigma_{8}=0.9$ and $h=0.73$. The Bower et al. model parameters were fixed with reference to a subset of the available observations of galaxies, mostly at low redshift. For further details we refer the reader to Bower et al. (2006). This model has previously been used for studying the clustering of galaxies at both low $(z \sim 0.1$, Kim et al. 2009) and high redshifts $(z \sim 1$, Gonzalez-Perez et al. 2011).

We note that GALFORM uses a Kennicutt IMF, whilst the photometric masses derived from the WIRDS data assume a Chabrier IMF. Based on Ilbert et al. (2010) and Gilbank et al. (2011), we multiply the GALFORM masses by a factor of 1.32 to match the Chabrier based stellar masses of the WIRDS photometric catalogues.

The Bower et al. model successfully reproduces the stellar mass function up to $z=4.5$ and the number counts of red galaxies at $z<2$ (Almeida et al. 2008; Gonzalez-Perez et al. 2009). In addition, we have shown in Bielby et al. (2012), that the mass function for all galaxies is well matched between our observations and the GALFORM model up to a redshift of at least $z \approx 2$.

\section{Galaxy clustering}

\subsection{The angular correlation function}

The angular correlation function can be measured using a number of estimators. In this paper, we use the Landy-Szalay estimator, which is given by:

$w(\theta)=\frac{\langle D D\rangle-2\langle D R\rangle+\langle R R\rangle}{\langle R R\rangle}$

where $\langle D D\rangle$ is the number of galaxy-galaxy pairs, $\langle D R\rangle$ is the number of galaxy-random pairs and $\langle R R\rangle$ is the number of random-random pairs. The Landy-Szalay estimator avoids the issue of linear biases seen in the direct estimator caused by spurious signal between the data and survey window. The random catalogues used to evaluate the estimator are produced using the survey geometry with the optical and NIR masks described above applied. The random galaxy catalogues each contain a total number of random data points equal to $20 \times$ the number of galaxies in the "real-data" catalogue with which the correlation function is being calculated.

As discussed, each of our fields measures $\approx 0.4-0.8 \mathrm{deg}^{2}$. Given these sizes, our data is subject to a bias in which the $w(\theta)$ estimator is biased low compared to the true correlation, given by:

$\sigma^{2}=\frac{1}{\Omega^{2}} \iint w(\theta) \mathrm{d} \Omega_{1} \mathrm{~d} \Omega_{2}$

where $\Omega$ is the areal coverage of the data. The bias, known as the Integral Constraint (e.g. Groth \& Peebles 1977; Peebles 1980; Roche et al. 1993; Baugh et al. 1996), results from estimating 
the mean density from the sample itself. Sampling larger areas reduces this effect, however it remains significant for the size of our survey fields. The "true" $w(\theta)$ is therefore given by:

$w(\theta)=\left\langle w_{\text {meas }}(\theta)\right\rangle+\sigma^{2}$

where $\left\langle w_{\text {meas }}(\theta)\right\rangle$ is the measured correlation function, averaged across the observed fields, and $w(\theta)$ is the correct correlation function. As in Roche et al. (2002), we evaluate the integral constraint using the numbers of random-random pairs in our fields:

$\sigma^{2}=A \frac{\sum N_{\mathrm{RR}}(\theta) \theta^{1-\gamma}}{\sum N_{\mathrm{RR}}(\theta)}$.

For the purposes of this work, we use the commonly used approach of fitting $w(\theta)$ results with a basic power-law of the form:

$w(\theta)=A \theta^{1-\gamma}$

with the separation angle, $\theta$ in arcminutes. We note that the characterization of galaxy clustering is more and more being treated using the halo-model approach (e.g. Hamana et al. 2004; Zheng et al. 2005; Brown et al. 2008; Wake et al. 2008). We restrict the analysis presented here to the power-law fitting, leaving a full halo-modelling analysis to future work. This is motivated by wishing to analyse the clustering in discrete bins by stellar mass, whereas a full halo model treatment would require the analysis to be performed using all galaxies above threshold mass limits. In this way, we may analyse the low mass galaxies in isolation in order to fully probe clustering trends with stellar mass. For the real-space clustering we assume the usual power-law form given by:

$\xi(r)=\left(\frac{r_{0}}{r}\right)^{\gamma}$

where $\xi$ is the real-space two-point correlation function (Peebles 1980) and $r$ is the real-space separation between two points. $r_{0}$ is then the characteristic separation and $\gamma$ is the power-law slope and is equivalent to the $\gamma$ in Eq. (5). We fit for the real-space clustering using the analytical transformation from $\xi(r)$ to $w(\theta)$ given by, for example, Phillipps et al. (1978), Peebles (1980), Simon (2007). We note that we do not use Limber's approximation, but use the full form (although the approximation makes little difference given the broadness of the redshift ranges considered when compared to the on-sky coverage).

It is also instructive to estimate the galaxy clustering bias, $b_{\mathrm{g}}$, from the clustering results. This gives the relationship between the clustering of the tracer population, i.e. the selected galaxy samples $\xi_{\mathrm{g}}(r)$, and the underlying dark matter clustering, $\xi_{\mathrm{DM}}(r)$.

$b_{\mathrm{g}}^{2}=\frac{\xi_{\mathrm{g}}(r)}{\xi_{\mathrm{DM}}(r)}$.

We estimate the bias by evaluating the integrated correlation function for both the galaxy clustering and the dark matter halo clustering, which is given by:

$\bar{\xi}\left(r_{\max }\right)=\frac{3}{r_{\max }^{3}} \int_{0}^{r_{\max }} \xi(r) r^{2} \mathrm{~d} r$.

For the purposes of this study, we use a value of $r_{\max }=$ $20 h^{-1} \mathrm{Mpc}$, which is a large enough scale to apply such that linear theory applies.

Prior to showing the results of the galaxy clustering analysis, it is important to note that the D2/COSMOS field presents some problems to such work. It is relatively well known that the COSMOS field contains an over-abundance of clusters at $z \sim 0.7$, which has a significant impact on galaxy clustering results. The cluster population in COSMOS and the overabundance at $z=0.7$ has been touched on by a number of papers (e.g. Guzzo et al. 2007; McCracken et al. 2007; Brusa et al. 2010; Skibba et al. 2014). Having four fields to study, we have the benefit of being able isolate this effect and upon doing so, have decided to exclude the D2 field in the redshift bin in which it is a strong outlier (i.e. $0.6<z<0.8$ in the analysis that follows). For completeness, we show the effect of the COSMOS field on the clustering measurement in the Appendix.

\subsection{Clustering of galaxies up to $z \sim 2$}

We first analyse the galaxy population split into bins of mass and redshift. The angular correlation functions are presented in Fig. 2, with each panel giving the results for a different redshift range as marked. In each panel, the triangles show the $10^{8.6}<$ $M\left[h^{-1} M_{\odot}\right] \leq 10^{9.6}$ sample, the squares the $10^{9.6}<M\left[h^{-1} M_{\odot}\right] \leq$ $10^{10.6}$ range, the $\times$ the $10^{10.6}<M\left[h^{-1} M_{\odot}\right] \leq 10^{11}$ range and the circles the $M\left[h^{-1} M_{\odot}\right]>10^{11}$ sample. Note that the correlation function is only plotted where sufficient galaxies in the given bin were present (i.e. $\gtrsim 150 \mathrm{deg}^{-2}$ ). Each $w(\theta)$ measurement represents the mean of the four fields, whilst the error estimates were calculated using a bootstrap analysis using 100 bootstrap resamplings. In all cases the points include the integral constraint contribution to the clustering.

We perform the power-law fitting to each measurement of the angular correlation function using the form given by Eq. (5). The fitting is limited to comoving separations of $r>1.2 /(1+$ z) $h^{-1} \mathrm{Mpc}$ comoving (given by the vertical dotted lines in each panel in Fig. 2) in order to minimise the contribution of the nonlinear regime (following the example of Foucaud et al. 2010). The resulting $A_{w}-\gamma$ fits are given by the dashed lines passing the points in Fig. 2 and the parameters, $A_{w}$ and $\gamma$ are provided in full in Table C.1. These power-law profiles successfully fit the data very well within the errors over the scales considered.

From Fig. 2, a clear link between clustering strength and galaxy stellar mass is evident, with more massive samples showing stronger clustering at all redshifts where multiple samples have been studied. We note that some of the higher mass clustering results (i.e. at $10^{10.6}<M\left[h^{-1} M_{\odot}\right] \leq 10^{11}$ ) show some signs of a break indicative of the 2-halo term.

In order to gain a clearer picture of the dependency of the clustering of the galaxy populations on mass and redshift we now estimate the real-space clustering properties of the populations using a single power-law prescription for the real-space correlation function as described in Sect. 3.1. We therefore determine the clustering length, $r_{0}$, and the slope, $\gamma$, for each stellar mass and redshift combination (again limiting the fits to just those points at separations of $r>1.2 /(1+z) h^{-1} \mathrm{Mpc}$ to minimise the impact of non-linear small scale clustering). The results are given in Table C.2, with quoted errors estimated from the bootstrap analysis.

We show the dependency of $r_{0}^{\gamma}$ on redshift and mass in Fig. 3. The symbols here are the same as in Fig. 2. The power-law fits affirm the significance of the observed link between stellar mass and the clustering strength across the redshift range. In addition, the $r_{0}^{\gamma}$ measurements now illustrate how the clustering evolves with redshift, showing that for a given mass, no significant evolution is seen in $r_{0}^{\gamma}$ with redshift up to $z \sim 2$. For comparison, we show results from Foucaud et al. (2010, grey crosses and asterisks) and Wake et al. (2011, grey stars). 
R. M. Bielby et al.: The WIRCam Deep Survey. II.

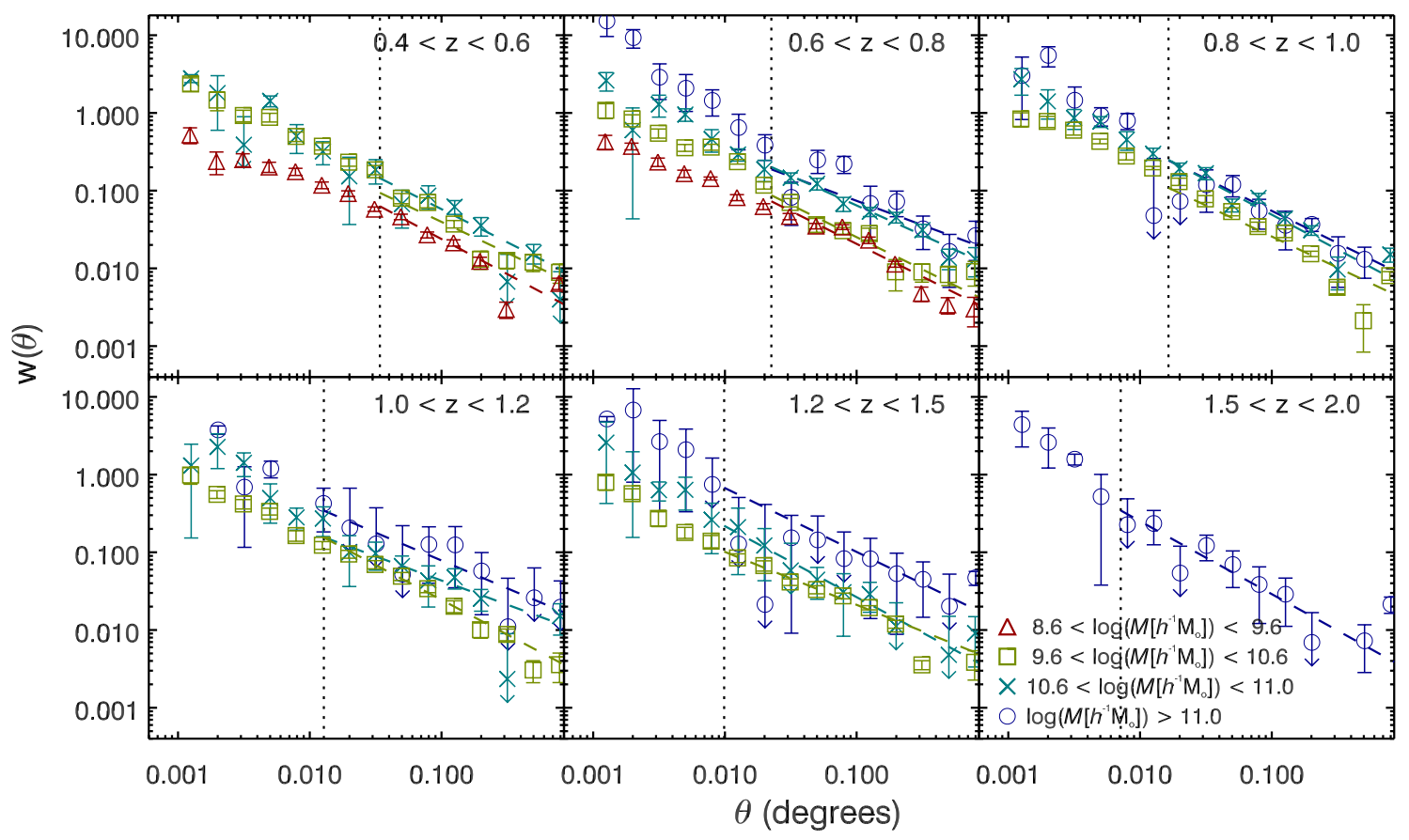

Fig. 2. Auto-correlation, $w(\theta)$, of all galaxies up to $z=2$ as a function of mass. The dotted vertical line in each figure gives the $\approx 1 h^{-1} \mathrm{Mpc}$ scale for the given redshift range above which the fits are made to the data (i.e. in the 2-halo term regime). The triangles give the $w(\theta)$ measurement for the lowest mass range, $10^{8.6}<M\left[h^{-1} M_{\odot}\right]<10^{9.6}$ range, the squares the $10^{9.6}<M\left[h^{-1} M_{\odot}\right]<10^{10.6}$ range, the $\times$ 's the $10^{10.6}<M\left[h^{-1} M_{\odot}\right] \leq 10^{11}$ range and the circles the $M\left[h^{-1} M_{\odot}\right]>10^{11.6}$ range. Errors were estimated using a bootstrap analysis and the dashed lines show the $A_{w}-\gamma$ power law fits.

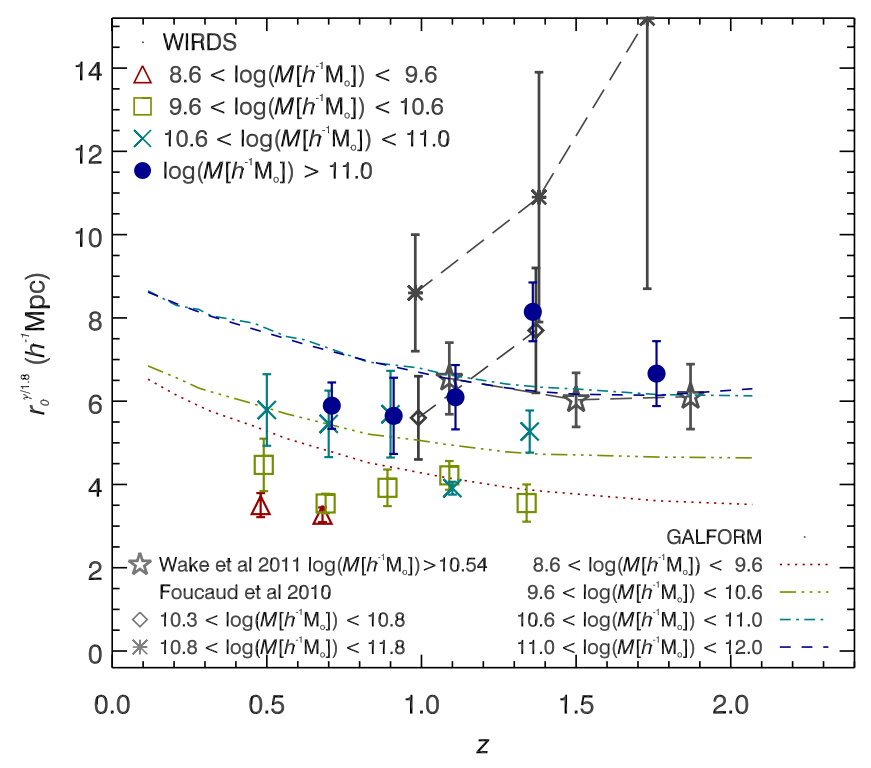

Fig. 3. Clustering strength, $r_{0}^{\gamma / 1.8}$ as a function of redshift for all galaxies with errors based on a bootstrap estimate. The populations are split by mass, with triangles showing the $10^{8.6}<M\left[h^{-1} M_{\odot}\right] \leq 10^{9.6}$ samples, squares $10^{9.6}<M\left[h^{-1} M_{\odot}\right] \leq 10^{10.6}$, ×'s $10^{10.6}<M\left[h^{-1} M_{\odot}\right] \leq 10^{11}$ and circles $M\left[h^{-1} M_{\odot}\right]>10^{11}$. The curves give the results of the GALFORM model for the observed mass ranges, as indicated in the legend. Results from Foucaud et al. (2010, grey asterisks and crosses connected by dashed lines) and Wake et al. (2011, grey stars connected by dashed lines) are also plotted.

The Foucaud et al. (2010) points show samples with mass ranges of $10^{10.5}<M\left[h^{-1} M_{\odot}\right]<10^{11.0}$ and $10^{11.0}<M\left[h^{-1} M_{\odot}\right]<$ $10^{12.0}$, which also show a significant mass dependence as with our results, but are reported as showing an increase in the clustering strength with redshift, at odds with our results. Indeed, the
Wake et al. (2011) measurements are more tightly constrained and agree well with our results, both in terms of the magnitude of the measured clustering and the overall trend with redshift of no significant redshift evolution in $r_{0}^{\gamma / 1.8}$. The WIRDS data is $\approx 1$ magnitude deeper than the Foucaud et al. (2010) data and covers approximately twice the area $\left(2.4 \mathrm{deg}^{2}\right.$ compared to $1.16 \mathrm{deg}^{2}$ ). The additional depth of the WIRDS data is particularly significant in the highest redshift bins, where we are likely probing a much greater proportion of the lower mass end of the $M>10^{11} M_{\odot}$ range than Foucaud et al. (2010).

Also plotted in Fig. 3 are the predictions from the GALFORM semi-analytic model described in Sect. 2.4, with each curve giving the clustering strength as a function of mass, as indicated in the legend. Comparing the observations with the GALFORM predictions, we find that the predicted mass dependence agrees with our observations. However, the GALFORM model marginally over-predicts the clustering when compared with the observations, except for the highest mass bins. The model also predicts an upturn in the clustering towards lower redshift (i.e. $z \lesssim 1$ ) since the modelled galaxies are basically following the dark matter halo trends (as shown for example in Foucaud et al. 2010 using Mo \& White 2002 models). This is not seen with any significance in the observations, however it can not be ruled out by them either.

Figure 4 shows the relation between the clustering strength and the space densities of these populations. The points show the results for the WIRDS galaxy samples, binned by mass identically as in previous plots, with the black diamonds showing measurements at $z=0.5$, blue triangles showing results for $z=0.7$, cyan squares the results for $z=0.9$, the green times-symbols $z=1.1$, the red stars $z=1.35$ and red crosses $z=1.75$. Number densities were calculated based on the galaxy stellar mass function calculated in the same redshift bins by Bielby et al. (2012). The dependence of clustering on galaxy space density is clear 


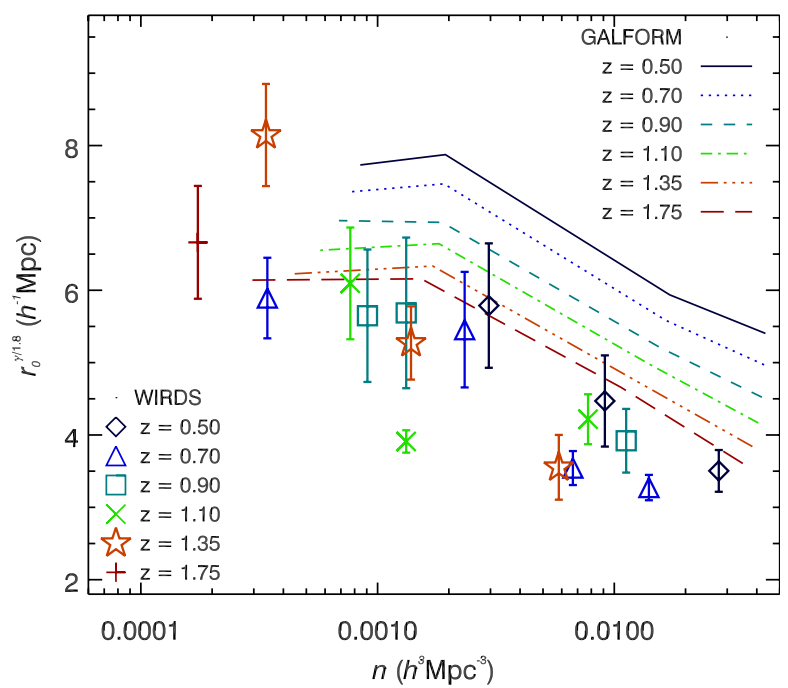

Fig. 4. Clustering strength, $r_{0}^{\gamma / 1.8}$ as a function of number density. The points show the results from the WIRDS analysis separated by redshift. The lines show the equivalent trends, again separated by redshift, from the GALFORM model. The trend of increasing clustering strength with decreasing space density is significant in the results.

and significant, with rarer more massive galaxies being more highly clustered. This is strongly connected to the stellar masses of the galaxy samples and the results presented in Fig. 3 where galaxy populations with higher masses are more strongly clustered. The trends predicted with the semi-analytical model are comparable to the observations. However the model predictions lie above those of the galaxy populations (whilst in Fig. 3 the simulations appeared to under-predict the clustering). Referring back to the analysis of the stellar mass functions in Bielby et al. (2012), this apparent conflict is clearly due to the over-prediction of galaxy number densities by the simulation. We also note that the model predicts a redshift evolution, with the trend moving to higher clustering strengths with lower redshift and that this trend with redshift is not seen in the observational results. This is particularly the case at high number densities $\left(n \gtrsim 0.004 h^{3} \mathrm{Mpc}^{-3}\right)$, where a tight line of data-points is seen compared to the evolution of the model curves. This appears to be at least in part driven by the over-prediction in the numbers of low mass galaxies seen in the galaxy stellar-mass functions presented in Bielby et al. (2012) for this same data and model combination.

\subsection{Clustering of galaxies by type}

We now perform the same analysis, but with the sample split into passive and star-forming galaxy populations. The sample is split based on derived dust-corrected rest-frame $N U V-r$ colours. The selection reliably differentiates galaxies based on star-formation rates in the sample to $z \sim 2$ and is described in detail by Ilbert et al. (2010) and Bielby et al. (2012). We apply an identical cut to the GALFORM model galaxies, producing measurements of the model galaxy clustering in an identical manner to that used for the observed galaxy populations.

The clustering results for passive and star-forming galaxies are shown in Figs. 5 and 6 respectively. We again fit the $w(\theta)$ measurements with $A_{w} \theta^{1-\gamma}$ power-laws, the results of which are plotted as dashed lines in Figs. 5 and 6 and recorded in Table C.1. Again the galaxy populations in each redshift range are split by mass, as indicated in the legend. The same trend of increasing clustering strength with increasing mass is seen for the star-forming population as in the full population. However, the results for the passive galaxies seem less clear, with the autocorrelations of the $10^{9.6}<M\left[h^{-1} M_{\odot}\right] \leq 10^{10.6}$ and $10^{10.6}<$ $M\left[h^{-1} M_{\odot}\right] \leq 10^{11}$ being largely consistent with each other in the redshift ranges where both are measured.

Again we calculate real-space correlation function powerlaw fits to the angular auto-correlation functions. The resulting $r_{0}$ and $\gamma$ parameters are given in Table C.2 and plotted in Fig. 7, with passive galaxies plotted in the left hand panel and star-forming in the right hand panel. It is clear from both the plots and the parameter values that, for a given mass bin and redshift, the passive galaxy samples are significantly more clustered than the star-forming galaxy populations. This dependency has been well probed at $z \lesssim 1$ (e.g. Davis \& Geller 1976; Phillipps et al. 1981; Loveday et al. 1995; Norberg et al. 2002), but results remain ambiguous at $z \gtrsim 1$ (e.g. Meneux et al. 2006; Coil et al. 2008; Hartley et al. 2010). Our observations are consistent with passive galaxies being more likely to exist in dense environments such as clusters and groups across the entire redshift range probed here (i.e. to $z=2$ ), with the star-forming populations having clustering strengths of $r_{0}^{\gamma} \lesssim 5 h^{-1} \mathrm{Mpc}$ (consistent with comparable measures made at $z \lesssim 1$, e.g. Norberg et al. 2002; Blake et al. 2009; Bielby et al. 2010a). This suggests that the process that has produced this difference in the clustering of passive and star-forming galaxies has already been at work much before $z \sim 2$.

We note however, that the most massive star-forming galaxies are seen to have clustering strengths of $r_{0}^{\gamma / 1.8} \sim 7-8 h^{-1} \mathrm{Mpc}$, approaching the clustering strengths of the passive galaxy samples. This is a similar result as that seen by Hartley et al. (2010), whereby they report clustering amplitudes for starforming galaxies (with absolute $K$-band magnitudes of $M_{K} \sim$ $-25)$ at $z \gtrsim 1.5$ comparable to those of passive galaxy samples. Hartley et al. (2010) suggest that they effectively see an increase indicative of star-forming galaxies being found in more highly clustered environments at high-redshift. Indeed, the authors conclude that the clustering strengths of star-forming galaxies decline towards $z=0$ for a given $K$-band luminosity (where this is a proxy for stellar mass). Our results are consistent with the clustering being constant with redshift for even for our highest mass bin (with some scatter due to the limited field size). We note also that the massive star-forming galaxies being as highly clustered as the passive population is consistent with the results of Tinker et al. (2013) at $z \lesssim 1$ in which it is seen that starforming galaxies above a mass limit of $M_{\star} \approx 10^{10.8} M_{\odot}$ have large scale clustering amplitudes comparable with those of the passive galaxy population. Significantly, our results provide new evidence for the continuation of this trend to $z \gtrsim 1$.

In Fig. 7, we plot results from a number of other studies of the clustering of star-forming galaxies across the redshift range we consider. The open circles show the clustering of photometrically selected star-forming galaxies at low redshift (Bielby et al. 2010a), the filled squares show comparable results from the DEEP2 survey presented by Coil et al. (2008) at $z \sim 1$ and the open stars show results for star-forming $B z K$ selected galaxies in the COSMOS field presented by McCracken et al. (2010).

Relating these magnitude selected samples to our massselected samples, we turn to the DEEP2 measurements of Cooper et al. (2008) that show a strong correlation between stellar mass and $M_{\mathrm{B}}$ absolute magnitude (with some additional correlation with $U-B$ colour). We have therefore estimated stellar mass ranges for the Bielby et al. (2010a) and Coil et al. (2008) galaxy samples based on Cooper et al. (2008). The $M_{\mathrm{B}} \sim-19.4$ and $M_{\mathrm{B}} \sim-20.2$ samples of Bielby et al. (2010a) correspond to median stellar masses of $M_{\star} \sim 10^{9.5} h^{-1} \mathrm{Mpc}$ 
R. M. Bielby et al.: The WIRCam Deep Survey. II.

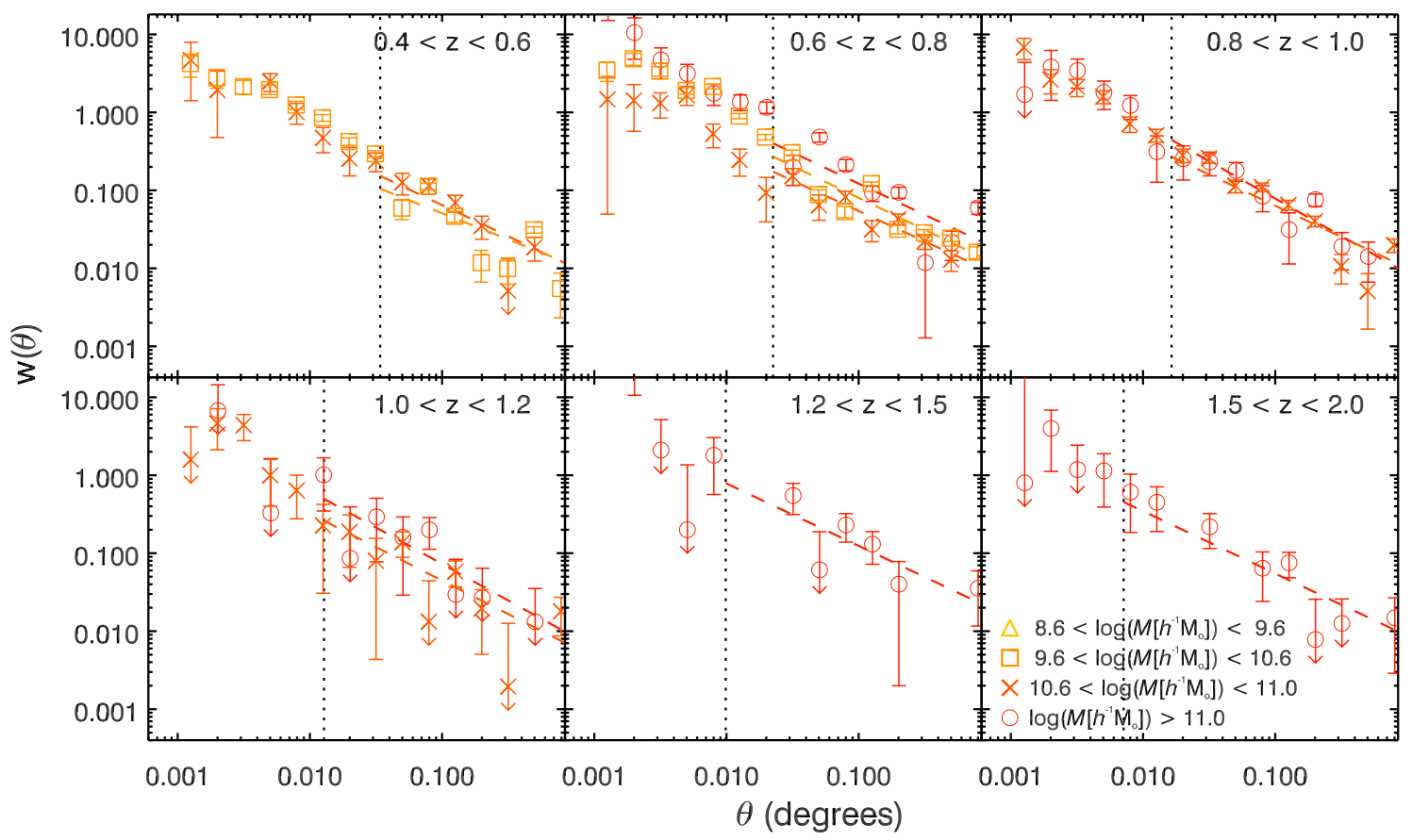

Fig. 5. As in Fig. 2, but for only the passive galaxy population selected by rest-frame dereddenned $M_{\mathrm{NUV}}-M_{r}$ colour.

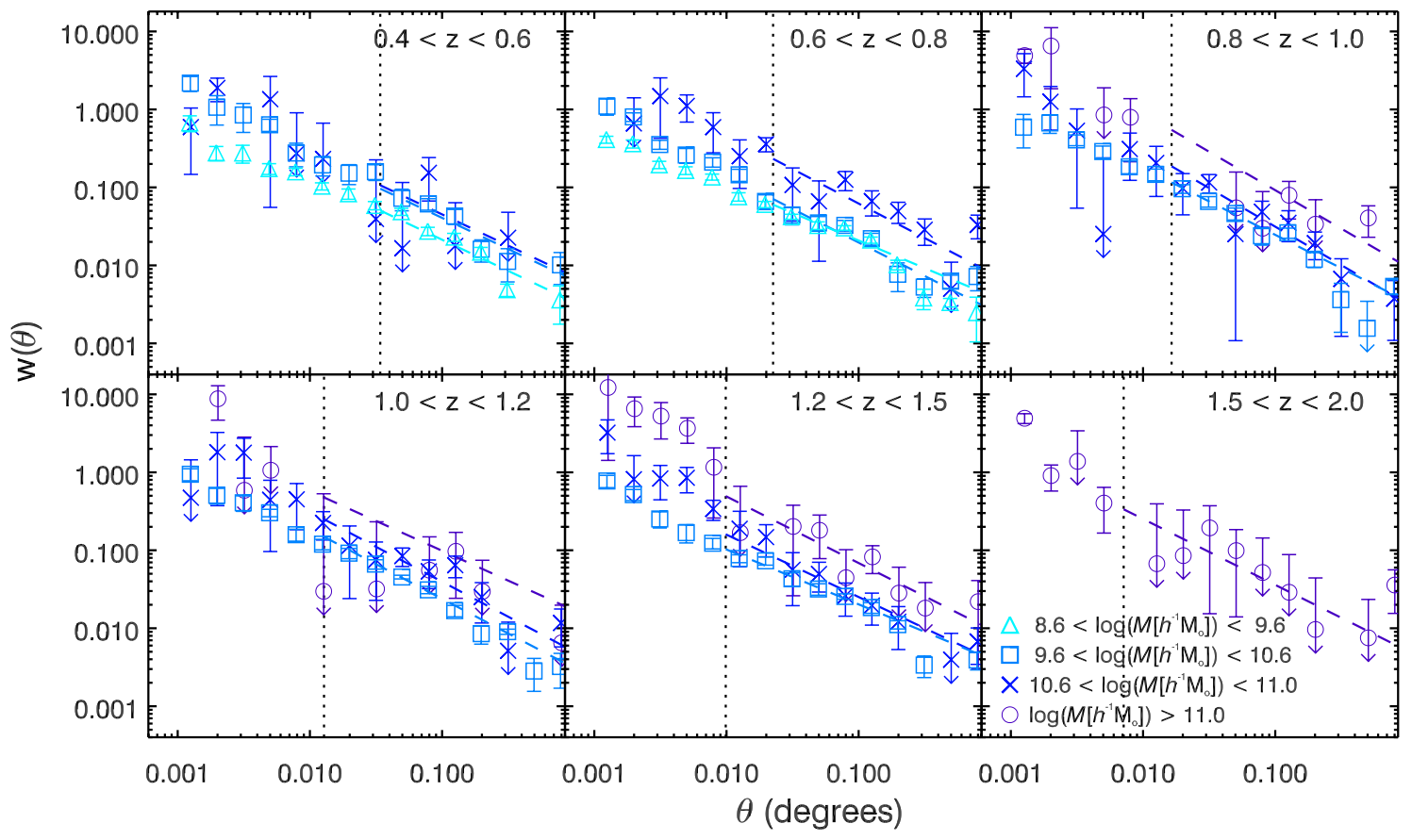

Fig. 6. As in Fig. 2, but for only the star-forming galaxy population selected by rest-frame dereddenned $M_{\mathrm{NUV}}-M_{r}$ colour.

and $M_{\star} \sim 10^{10} h^{-1} \mathrm{Mpc}$ respectively, whilst the $M_{\mathrm{B}}<-20$ and $M_{\mathrm{B}}<-21$ samples of Coil et al. (2008) correspond to $M_{\star} \gtrsim 10^{10} h^{-1} \mathrm{Mpc}$ and $M_{\star} \gtrsim 10^{10.4} h^{-1} \mathrm{Mpc}$. In addition, we have estimated the stellar mass ranges for the sBzK selections of McCracken et al. (2010), using our own D2/COSMOS data. For the star-forming BzK galaxies, we find stellar mass ranges of $M_{\star} \gtrsim 10^{9.6} h^{-1} \mathrm{Mpc}$ and $M_{\star} \gtrsim 10^{10.1} h^{-1} \mathrm{Mpc}$ for the $K<23$ and $K<22$ mag cuts respectively.

These samples add to the picture of little overall change in the relationship between clustering strength of star-forming galaxies and mass over time since $z \sim 2$. Indeed, results for spectroscopic samples of LBGs at $z \sim 3$, which are broadly representative of the luminous star-forming population, give clustering lengths of $r_{0}^{\gamma / 1.8} \sim 4 h^{-1} \mathrm{Mpc}$ (e.g. Adelberger et al. 2003, 2005; Bielby et al. 2011, 2013). These are estimated to have mean stellar masses of $M_{\star} \sim 10^{10.3} h^{-1} M_{\odot}$ (Shapley et al. 2003) at which mass we measure clustering lengths of $3.8 h^{-1} \mathrm{Mpc}<r_{0}<4.5 h^{-1} \mathrm{Mpc}$, adding to our observation that there is little evolution in the clustering as measured via $r_{0}^{\gamma / 1.8}$ as a function of redshift to $z \sim 2-3$.

Returning to the passive galaxies, all but two of the observational points agree at the $1 \sigma$ level with the GALFORM predictions. Both observations and the model predictions suggest little change in clustering strength with redshift. This lack of 


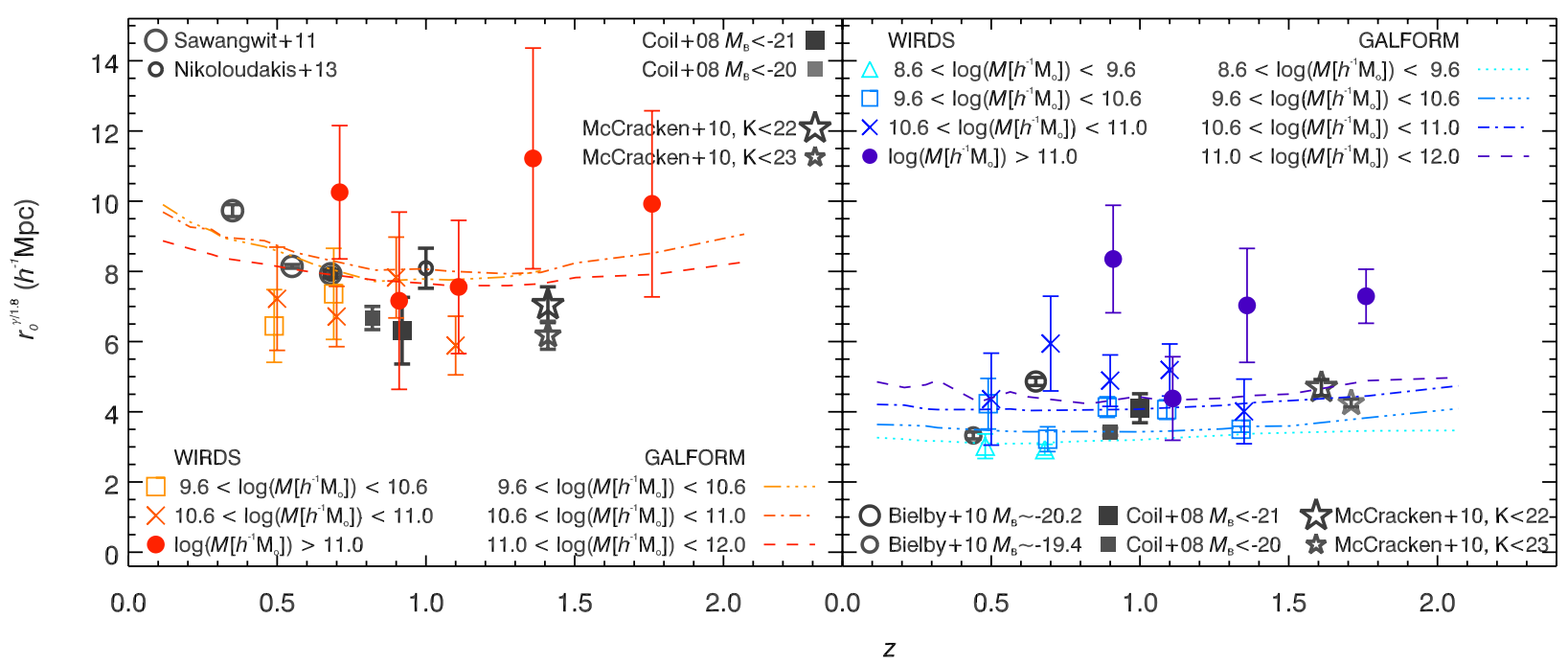

Fig. 7. Clustering strength, $r_{0}^{\gamma / 1.8}$ as a function of redshift for passive galaxies (left panel) and star-forming galaxies (right panel). In each case, the populations are split by mass, with triangles showing the $10^{8.6}<M\left[h^{-1} M_{\odot}\right] \leq 10^{9.6}$ range, squares $10^{9.6}<M\left[h^{-1} M_{\odot}\right] \leq 10^{10.6}, \times^{\prime} \mathrm{s} 10^{10.6}<$ $M\left[h^{-1} M_{\odot}\right] \leq 10^{11}$ and circles $M\left[h^{-1} M_{\odot}\right]>10^{11}$. The curves give the predictions of the GALFORM model for the different mass bins as indicated in the legend.

evolution with redshift is consistent with previous results at lower redshift. In the redshift range $0.4 \lesssim z \lesssim 1.0$, LRG observations (of samples of approximately uniform absolute magnitude) are consistent with no evolution of $r_{0}^{\gamma / 1.8}$ as a function of redshift. In Fig. 7, we show the LRG clustering amplitudes of Sawangwit et al. (2011) and Nikoloudakis et al. (2013) by the large and small circles respectively. Based on their absolute $i$-band magnitudes, these LRG samples have stellar mass ranges of $M_{\star} \gtrsim$ $10^{10.4} h^{-1} M_{\odot}$, except for the $z=0.35$ point of Sawangwit et al. (2011) which corresponds to $M_{\star} \gtrsim 10^{10.7} h^{-1} M_{\odot}$. The WIRDS data are consistent with the overlapping $z \gtrsim 0.4 \mathrm{LRG}$ results, but extend this conclusion to lower mass samples than the LRGs and to higher redshift.

We also plot the clustering amplitudes for the passive galaxy samples of Coil et al. (2008) and the passive BzK selection of McCracken et al. (2010). As with the equivalent star-forming populations discussed above, we derive stellar mass ranges for each of these. The $M_{\mathrm{B}}<-20$ and $M_{\mathrm{B}}<-21$ samples of Coil et al. (2008) correspond to $M_{\star} \gtrsim 10^{10.5} h^{-1} \mathrm{Mpc}$ and $M_{\star} \gtrsim$ $10^{10.8} h^{-1} \mathrm{Mpc}$ respectively, whilst the $K<23$ and $K<22$ of McCracken et al. (2010) correspond to $M_{\star} \gtrsim 10^{10.6} h^{-1} \mathrm{Mpc}$ and $M_{\star} \gtrsim 10^{10.7} h^{-1} \mathrm{Mpc}$. Again, we see for all these samples with comparable mass ranges spread over a large redshift range, little evidence for any evolution in the clustering amplitude with redshift for a given mass.

In terms of the stellar mass dependence of the clustering for passive galaxies, as stated we find that the data show no significant correlation. This is similar to the results reported by Hartley et al. (2013) for clustering with stellar mass and Williams et al. (2009) for clustering as a function of luminosity for passive galaxies. In fact we note that over the absolute luminosity range we probe with our samples (i.e. $-16 \gtrsim M_{g} \gtrsim-22$ ), this parallels previous work at low redshift. In particular, Norberg et al. (2002) measured the clustering of the passive population as a function of absolute $B_{j}$-band magnitude and found that the clustering results showed little dependence on absolute magnitude, at least at $M_{\mathrm{bj}} \gtrsim-21-5 \log h$. Brighter than $M_{\mathrm{bj}} \sim-21-5 \log h$, Norberg et al. (2002) did find that the clustering results increased with brighter $M_{\mathrm{bj}}$, however again taking the results of Cooper et al. (2008), $M_{\mathrm{bj}}$ corresponds to a stellar mass of $\sim 10^{11} h^{-1} M_{\odot}$. This is equivalent to our highest mass bin and although the clustering results are elevated in this mass bin, the measured uncertainties on these high mass points preclude any detection of a clear dependency at high mass.

Further to this, significant correlations between clustering strength and luminosity are reported for higher luminosity systems (e.g. Sawangwit et al. 2011). Combining our own results with Hartley et al. (2013) and the lower redshift results, we conclude that similarly to the luminosity dependence shown by Norberg et al. (2002), the clustering of passive galaxies has little dependence on stellar mass below masses of $M_{\star} \lesssim 10^{11} h^{-1} M_{\odot}$, but are unable to constrain any possible dependency on stellar mass above this limit. The GALFORM model also predicts only a very small dependency of $r_{0}^{\gamma / 1.8}$ on $M_{*}$ for passively evolving galaxies. This is found to be the result of the passive galaxy host halo mass being, on average, a constant with the predicted galaxy stellar mass.

The result of an observed red-sequence with high clustering levels to $z \approx 2$ is complimentary and consistent with observations of the red-sequence to be in place in galaxy clusters to such redshfits (e.g. Tanaka et al. 2010; Gobat et al. 2011) and the lack of evolution in these red-sequence galaxies and brightest cluster galaxies (e.g. Onodera et al. 2010; Stott et al. 2011; Bielby et al. 2012).

The results for the star-forming populations are shown in the right-hand panel of Fig. 7. Here again we see little sign of evolution in the clustering strength over the redshift range probed. As discussed, the most massive star-forming galaxies at $z>1.2$ do lie in strongly clustered regions and have clustering strengths comparable to the passive galaxy population. We note that this epoch coincides with recent claims of significant star-formation rates in high redshift clusters. For example, Hilton et al. (2010) report observations of $24 \mu \mathrm{m}$ sources within $<250 \mathrm{kpc}$ of the centre of the high redshift cluster XMMXCS J2215.9-1738 $(z=1.46)$, which they report suggests that a large amount of star formation may be taking place in the cluster core, in contrast to clusters at lower redshifts. Similarly, Tran et al. (2010) measure an increase in the fraction $24 \mu \mathrm{m}$ luminous star-forming galaxies towards the centre of the $z=1.62$ cluster CIG J0218.3-0510, again in contradiction to results at lower redshift and signifying 
R. M. Bielby et al.: The WIRCam Deep Survey. II.

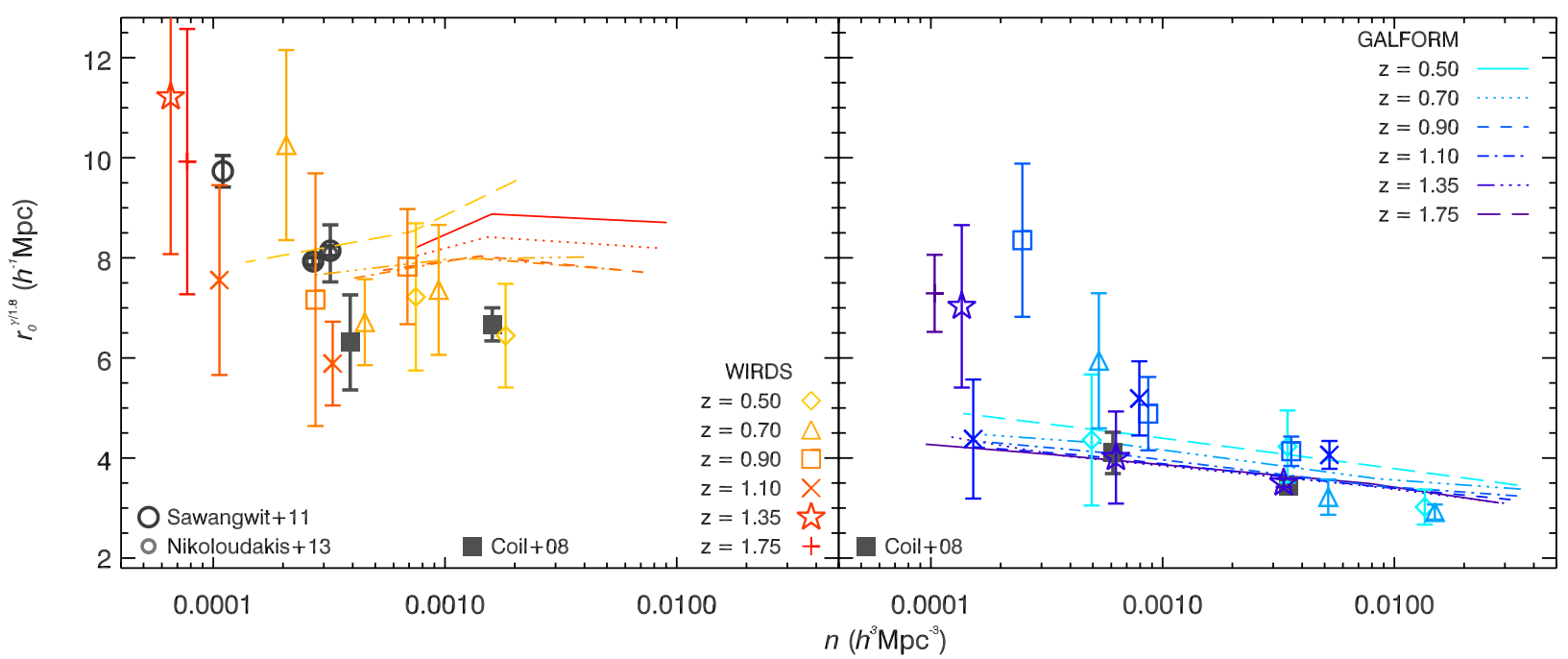

Fig. 8. Clustering strength, $r_{0}^{\gamma / 1.8}$ as a function of number density, split by galaxy type, with passive galaxies shown in the left hand panel and star-forming galaxies in the right hand panel. In both panels, the coloured symbols show the results for the WIRDS data, whilst the lines show the predictions from GALFORM. The grey symbols show results from the literature, with the $z=0.3,0.5,0.7 \mathrm{LRG}$ results from Sawangwit et al. (2011, large open circles), the $z=0.7,1.0 \mathrm{LRG}$ results from Nikoloudakis et al. (2013, small open circles) and the passive (plotted left) and star-forming (plotted in the right panel) $z=1$ results of Coil et al. (2008, filled squares in both panels).

a shift in the location of star-formation to the high density regions.

We see good agreement between the observations and the model, with both showing relatively constant clustering strengths as a function of redshift for each mass range. We also observe a stronger mass dependence at all redshifts for the starforming populations than for the passive galaxies. This is again the case for both the observational results and the GALFORM model predictions. For clarity we note that the lowest dashed line corresponds to the same mass range as the triangular points, the second line to the square points, the third to the $x$ and the highest to the mass range of the filled circles. The star-forming population therefore appears to be the dominant contributor to the increase in clustering with galaxy stellar mass content, whilst the passive galaxies show a smaller, less pronounced, change in clustering with stellar mass within the mass ranges we are probing.

In Fig. 8, we show the clustering results for the passive (left) and star-forming (right) galaxies as a function of sample number density. In each case the points are split by redshift, with the diamonds corresponding $z=0.5$, the triangles to $z=0.7$, the squares to $z=0.9$, the times symbols to $z=1.1$, the filled circles to $z=1.35$ and the crosses to $z=1.75$. Interestingly, the GALFORM predictions suggest little dependence of clustering strength on galaxy number density for the passive galaxies. This is closely related to the small galaxy stellar mass dependence seen in the previous figure. Using the WIRDS data alone it is difficult to constrain this prediction, however we can add in results from previous work to aid the analysis. As such we include the previous points for LRGs, which represents a strongly clustered low number-density population, and the passive galaxies analysed by Coil et al. (2008). These are consistent with the WIRDS points, with the Coil et al. (2008) points in particular corroborating the lack of evolution seen in the GALFORM predictions, whilst the Sawangwit et al. (2011) results suggest some dependence of $r_{0}^{\gamma / 1.8}$ at number densities of $\lesssim 3 \times 10^{-4} h^{3} \mathrm{Mpc}^{-3}$.

A more clear variation of $r_{0}^{\gamma / 1.8}$ is seen with number density in the right hand panel, where the results are shown for star-forming galaxies. In comparison to the passive population, far less scatter is seen in the results, due to the larger numbers of star-forming galaxies and the resulting improvement in statistical errors. Again we show points from Coil et al. (2008) and find good agreement between their results for star-forming galaxies and our own. The GALFORM model predicts a relation between $r_{0}^{\gamma / 1.8}$ and number density that is consistent with the WIRDS results down to number densities of $\sim 2 \times 10^{-4} h^{3} \mathrm{Mpc}^{-3}$, where the observational data suggest a potential upturn in the trend.

\subsection{Dark matter halo mass}

From the clustering results, we may estimate the mean masses of dark matter halos within which the galaxy samples reside. We now do this using the formalism developed by Mo \& White (1996). This provides a relationship between the bias of galaxy clustering to the mean halo mass, based on a model of spherical collapse and tested with N-body simulations. An extension to the method was made based on ellipsoidal collapse by Sheth et al. (2001), which relates the halo-bias to the mean halo mass via:

$$
\begin{aligned}
b_{\mathrm{h}}\left(M_{\mathrm{DM}}, z\right)= & 1+\frac{1}{\sqrt{a} \delta_{\mathrm{c}}}\left[a v^{2} \sqrt{a}+b \sqrt{a}\left(a v^{2}\right)^{1-c}\right. \\
& \left.-\frac{\left(a v^{2}\right)^{c}}{\left(a v^{2}\right)^{c}+b(1-c)(1-c / 2)}\right]
\end{aligned}
$$

where $a, b$ and $c$ are constants for which we take the values given by Tinker et al. (2005): $a=0.707, b=0.35$ and $c=0.8$. $\delta_{\text {c }}$ is the critical overdensity required for collapse and is given by $\delta_{\mathrm{c}}=0.15(12 \pi)^{2 / 3} \Omega_{\mathrm{m}}(z)^{0.005} \approx 1.686$ (Navarro et al. 1997). The variable $v$ is defined as $\delta_{\mathrm{c}} / \sigma\left(M_{\mathrm{DM}}, z\right)$, where $\sigma\left(M_{\mathrm{DMH}, z}\right)$ is the rms fluctuation of the density field and can be separated into dark matter halo mass and redshift dependancies via $\sigma\left(M_{\mathrm{DM}}, z\right)=$ $\sigma\left(M_{\mathrm{DM}}\right) D(z)$. Here $D(z)$ is the linear growth rate and the mass dependence of the rms fluctuation is given by:

$$
\sigma\left(M_{\mathrm{DM}}\right)^{2}=\frac{1}{2 \pi^{2}} \int_{0}^{\infty} k^{2} P(k) w(k r)^{2} \mathrm{~d} k .
$$

Here, $P(k)$ is the matter power-spectrum, which we calculate using CAMB (Lewis et al. 2000; Challinor \& Lewis 2011), which is 


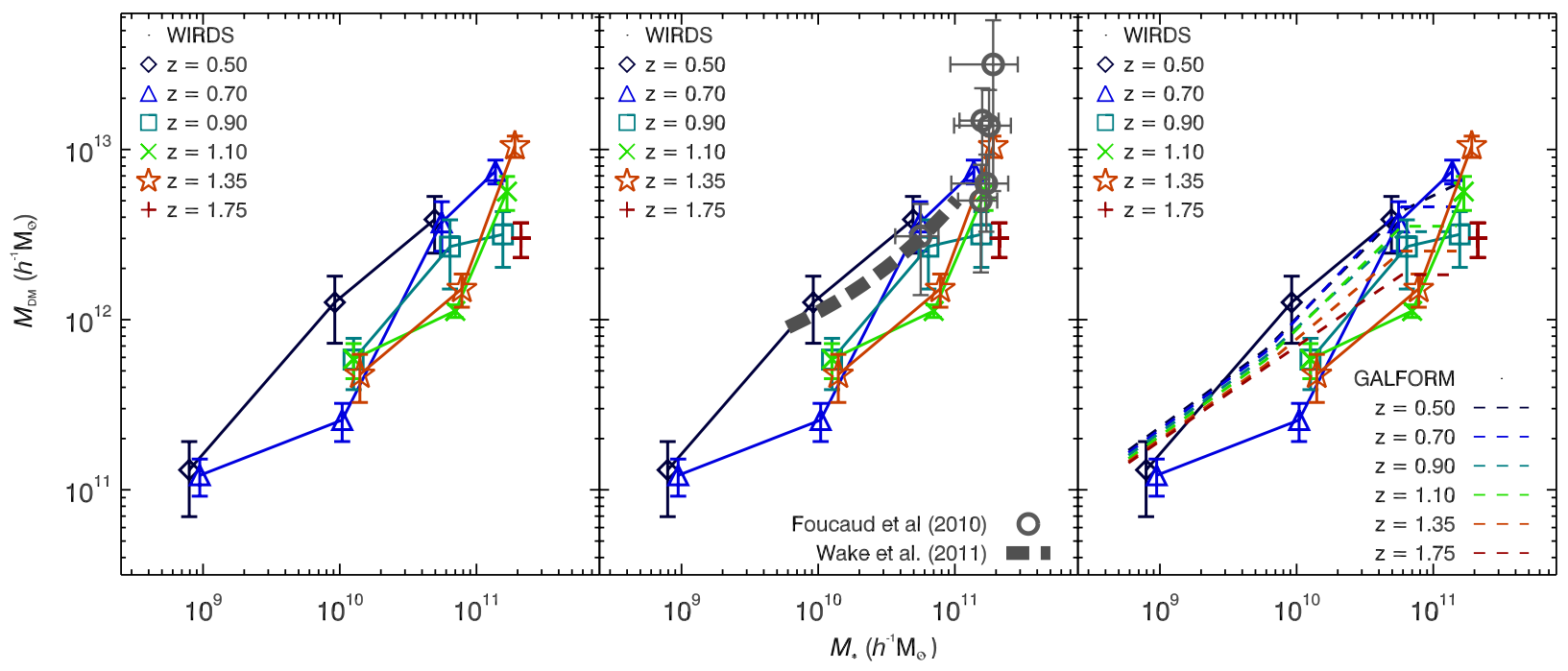

Fig. 9. Host dark matter halo mass, $M_{\mathrm{DM}}$, as a function of galaxy stellar mass, $M_{*}$ for the full galaxy sample split by mass and redshift. The WIRDS results are reproduced in all three panels, whilst literature results are added in the centre panel and the GALFORM predictions are added in the right hand panel. In terms of the WIRDS data, the triangles, squares, $\times$, stars and crosses show the results from the WIRDS data for redshift ranges centred on $z=0.50, z=0.70, z=0.90, z=1.10, z=1.35$ and $z=1.75$ respectively. In the centre panel, the thick dashed curve shows the fit to $M_{\mathrm{DM}}$ versus $M_{\star}$ given by Wake et al. (2011) for galaxies at $1<z<2$ in the NEWFIRM survey, whilst the open circles show the results from Foucaud et al. (2010) at $z \gtrsim 1$. In the right hand panel, the predictions from the GALFORM model are given for the same central redshifts.

based on CMBFAST (Seljak \& Zaldarriaga 1996; Zaldarriaga \& Seljak 2000). $w(k r)$ is the window function for a spherical tophat function given by:

$w(k r)=3 \frac{\sin (k r)-k r \cos (k r)}{(k r)^{3}}$

where $r$ is the top-hat radius and is related to the mass, $M_{\mathrm{DM}}$ by:

$r=\left(\frac{3 M_{\mathrm{DM}}}{4 \pi \rho_{0}}\right)^{\frac{1}{3}}$

$\rho_{0}$ is the present day mean density of the universe and is given by $\rho_{0}=\Omega_{\mathrm{m}}^{0} \rho_{\text {crit }}^{0}=2.78 \times 10^{11} \Omega_{\mathrm{m}}^{0} h^{2} M_{\odot} \mathrm{Mpc}^{-3}$.

Combining Eqs. (9)-(12) allows us to estimate the dark matter halo mass from the clustering bias, which we match to the calculated bias for each of the galaxy samples.

We show the results of the bias matching in Fig. 9, where we plot the median dark matter halo mass, $M_{\mathrm{DM}}$, versus the mean galaxy sample stellar mass, $M_{*}$. This is based on the full galaxy sample clustering as presented in Fig. 3. The WIRDS data, which is repeated in all three panels, is split by redshift with the black diamonds showing the $z=0.5$ points, the dark blue triangles showing the $z=0.7$ points, the blue squares showing the $z=0.9$ points, the cyan times symbols the $z=1.1$ points, the green stars the $z=1.35$ points and the red crosses the $z=1.75$ points. At each redshift, a clear dependence of $M_{\mathrm{DM}}$ on stellar mass is seen. In addition, there is some sign of a redshift evolution, with $M_{\mathrm{DM}}$ appearing to move lower for a given stellar mass with increasing redshift.

Comparing to equivalent datasets in the literature (centre panel), the grey circles show the points of Foucaud et al. (2010) and the long-dashed curve shows a fit from Wake et al. (2011). The Foucaud et al. (2010) points are based on equivalent redshift and mass bins to our highest redshift bins. As in Fig. 3, the WIRDS and Foucaud et al. (2010) results agree well except where the latter find large clustering results: in this case halo masses of $M_{\mathrm{DM}} \gtrsim 10^{13} h^{-1} \mathrm{Mpc}$. Our results at these masses and redshifts (green stars and red crosses) suggest much lower halo masses $M_{\mathrm{DM}} \gtrsim 4-8 \times 10^{12} h^{-1} \mathrm{Mpc}$. However, given the large error bars on the Foucaud et al. (2010) points, their results are consistent with those of WIRDS as well as the relation derived in Wake et al. (2011).

Now taking the GALFORM predictions (right hand panel), we find that these agree with the WIRDS results to within $\approx 1 \sigma$ of the data points across the different redshift ranges. Interestingly, we see that the GALFORM results predict a redshift evolution in the halo masses for a given stellar mass cut. The range covered by the WIRDS results and the associated errors limit our ability to confirm whether this is a genuine evolution. We also note that in the model, the relationship between $M_{\mathrm{DM}}$ and $M_{\star}$ is primarily driven by the star-forming population, whilst the halo masses of passive galaxies show little dependence on the stellar mass of the galaxies.

Taking our estimates for halo masses from the clustering results, we now plot these against number density, $n$, in Fig. 10. We use the same symbols for different redshift bins as in Fig. 9. Also plotted is the $n-M_{\mathrm{DM}}$ relation determined for low-redshift $(z \lesssim 1)$ galaxies by Coupon et al. (2012) from the CFHTLS Wide field data. Again, the GALFORM results are shown by the short dashed coloured lines, with the colours coordinated with the WIRDS data points (i.e. from black for low redshift to red for high redshift). Both here and for the halo mass as a function of stellar mass, this redshift evolution in the model is showing the galaxies occupying, on average, more massive dark matter halos at lower-redshift resulting from the general dark matter halo trends.

One point to note from the WIRDS data is that plotted in this way, the $z=0.7$ points are consistent with the $z=0.5$ and $z=0.9$ results. However, above $z \sim 1$, we now see a tentative trend for the WIRDS results to shift to lower halo masses for a given number density. This effect is also present in the GALFORM predictions, with the dashed lines moving down and to the left (note that the GALFORM results are based on the same stellar mass bins as the data points) with increasing redshift. 
R. M. Bielby et al.: The WIRCam Deep Survey. II.

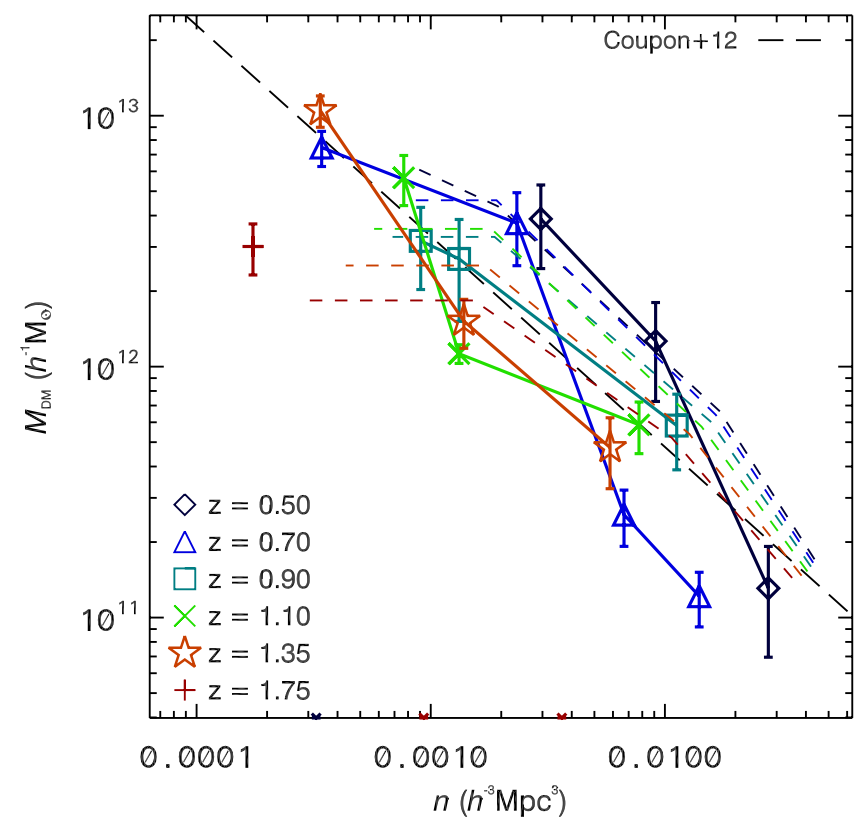

Fig. 10. Mean halo mass versus galaxy number density for our range of galaxy stellar mass and redshift ranges. The points show our results with different point types giving different redshift ranges. The long-dashed line shows the best fit determined by Coupon et al. (2012) for $z<1$ galaxies in the CFHTLS. The short-dashed curves show the GALFORM predictions with the same colour coding as given in Fig. 9.

In summary, from the halo mass analysis, we confirm the relationship between galaxy stellar mass and halo mass across the redshift range of $0.4 \lesssim z \lesssim 2.0$ in agreement with previous work, whilst finding tentative evidence for an evolution in the relationship between number density and halo mass.

\section{Conclusions}

Based on the deep 8-band CFHTLS+WIRDS photometry, we have conducted an analysis of clustering up to $z=2$, evaluating galaxy spatial correlations as a function of mass and type. Complimentary to this, we have used the GALFORM semianalytical model of galaxy formation and evolution to make clustering predictions using identical selection constraints based on the same galaxy properties. From the WIRDS data, we find a constant clustering strength within the error estimates as a function of redshift over the redshift range $0.3<z<2$ for the full galaxy sample. At the same time we detect a mass dependence for clustering over the whole range such that galaxies with greater stellar masses are more strongly clustered, extending previous results to higher redshifts. The GALFORM model predictions are consistent with the results from the data.

Comparing the clustering between star-forming and passive galaxies, we again find that both remain constant in clustering strength within the errors as a function of redshift (in the range $0.3<z<2$ ) for a given mass limit. Thus the "passivity" dependence of clustering, such that passive galaxies are more strongly clustered than star-forming ones, is confirmed up to $z \approx 2$. This is only broken at high stellar mass $\left(M_{\star} \gtrsim\right.$ $\left.10^{11} h^{-1} M_{\odot}\right)$ at which point we find star-forming galaxies have comparable clustering (at the large scales probed) to passive galaxies. The GALFORM model predictions for the same mass and redshift constraints reproduce the observations well within the observational errors, although do not predict the high clustering found in the high mass star-forming galaxy population.
We find little dependence on mass for the clustering of the passive population across the stellar mass range covered here. This is consistent with the dependence with $b_{j}$-band magnitude of galaxies brighter than $M_{b j}<-21$ (Norberg et al. 2002, a range within which the majority of our galaxy mass samples are within). Taking the star-forming population, a stronger dependence of clustering strength on stellar mass is observed than for the passive galaxies, a trend that is seen in both the WIRDS data and the GALFORM model predictions.

Finally we have investigated the relation between galaxy stellar mass and mean dark matter halo mass for the samples described above. Based on the Mo \& White (1996) formalism, we have estimated mean dark matter halo masses from the clustering measurements for the full galaxy sample. We see a significant trend of mean halo mass increasing with galaxy stellar mass across a range of redshifts. Additionally, we find the tentative result that given a constant stellar mass the halo mass increases marginally with decreasing redshift.

The above results have built on the current picture of galaxy evolution via clustering analyses, adding to the previous works of Foucaud et al. (2007) and Wake et al. (2008) with deeper limits in magnitude and mass over an overall larger area. The key results that the passive and star-forming populations remain relatively constant in terms of clustering strength as a function of redshift up to $z \approx 2$ (given a constant mass selection) fits in well with complimentary observations showing little evolution in the stellar mass function over a similar range (Ilbert et al. 2010). It will now be interesting to build on these observations by pushing further down the stellar mass function at $z \gtrsim 1$ with deeper and wider NIR data from the upcoming surveys such as UltraVISTA, to constrain the clustering and hence galaxy evolution for galaxies across a broader range of stellar masses.

Acknowledgements. R.M.B. acknowledges the funding of the French Agence Nationale de la Recherche (ANR) and the UK Science and Technology Facilities Council. H.J.McC. acknowledges support from the "Programme national cosmologie et galaxies". This work is based in part on data products produced at TERAPIX at the Institut d'Astrophysique de Paris and the Canadian Astronomy Data Centre (CADC) as part of the Canada-France-Hawaii Telescope (CFHT) Legacy Survey, a collaborative project of the Canadian National Research Council (NRC) and Centre National de la Recherche Scientifique (CNRS). Much of the work is based on observations at the CFHT, which is funded by the NRC, the CNRS, and the University of Hawaii. This research has made use of the VizieR catalogue access tool provided by the CDS, Strasbourg, France. This research was supported by ANR grant "ANR-07-BLAN-0228". E.D. also acknowledges support from "ANR-08-JCJC-0008". J.P.K. acknowledges support from the CNRS. GALFORM was run on the ICC Cosmology Machine, which is part of the DiRAC Facility jointly funded by STFC, the Large Facilities Capital Fund of BIS, and Durham University. Finally, we thank the anonymous referee for her/his constructive and valuable comments.

\section{References}

Adelberger, K. L., Steidel, C. C., Shapley, A. E., \& Pettini, M. 2003, ApJ, 584, 45

Adelberger, K. L., Steidel, C. C., Pettini, M., et al. 2005, ApJ, 619, 697 Almeida, C., Baugh, C. M., Wake, D. A., et al. 2008, MNRAS, 386, 2145 Arnouts, S., Moscardini, L., Vanzella, E., et al. 2002, MNRAS, 329, 355 Arnouts, S., Walcher, C. J., Le Fèvre, O., et al. 2007, A\&A, 476, 137

Baugh, C. M. 2006, Rep. Prog. Phys., 69, 3101

Baugh, C. M., Gardner, J. P., Frenk, C. S., \& Sharples, R. M. 1996, MNRAS, 283, L15

Baugh, C. M., Lacey, C. G., Frenk, C. S., et al. 2005, MNRAS, 356, 1191 Benson, A. J., Bower, R. G., Frenk, C. S., et al. 2003, ApJ, 599, 38 Bielby, R., Shanks, T., Sawangwit, U., et al. 2010a, MNRAS, 403, 1261 Bielby, R. M., Finoguenov, A., Tanaka, M., et al. 2010b, A\&A, 523, A66 Bielby, R. M., Shanks, T., Weilbacher, P. M., et al. 2011, MNRAS, 414, 2 Bielby, R., Hudelot, P., McCracken, H. J., et al. 2012, A\&A, 545, A23 Bielby, R., Hill, M. D., Shanks, T., et al. 2013, MNRAS, 430, 425 
Blake, C., Jurek, R. J., Brough, S., et al. 2009, MNRAS, 395, 240 Blanc, G. A., Lira, P., Barrientos, L. F., et al. 2008, ApJ, 681, 1099 Bower, R. G., Benson, A. J., Malbon, R., et al. 2006, MNRAS, 370, 645 Brown, M. J. I., Jannuzi, B. T., Dey, A., \& Tiede, G. P. 2005, ApJ, 621, 41 Brown, M. J. I., Zheng, Z., White, M., et al. 2008, ApJ, 682, 937 Brusa, M., Civano, F., Comastri, A., et al. 2010, ApJ, 716, 348 Challinor, A., \& Lewis, A. 2011, Phys. Rev. D, 84, 043516 Coil, A. L., Newman, J. A., Cooper, M. C., et al. 2006, ApJ, 644, 671 Coil, A. L., Newman, J. A., Croton, D., et al. 2008, ApJ, 672, 153 Cole, S., Lacey, C. G., Baugh, C. M., \& Frenk, C. S. 2000, MNRAS, 319, 168 Connolly, A. J., Szalay, A. S., Dickinson, M., Subbarao, M. U., \& Brunner, R. J. 1997, ApJ, 486, L11

Conroy, C., Shapley, A. E., Tinker, J. L., Santos, M. R., \& Lemson, G. 2008, ApJ, 679, 1192

Cooke, J., Wolfe, A. M., Gawiser, E., \& Prochaska, J. X. 2006, ApJ, 652, 994 Cooper, M. C., Newman, J. A., Weiner, B. J., et al. 2008, MNRAS, 383, 1058 Coupon, J., Kilbinger, M., McCracken, H. J., et al. 2012, A\&A, 542, A5

Cowie, L. L., Gardner, J. P., Hu, E. M., et al. 1994, ApJ, 434, 114 Cucciati, O., Tresse, L., Ilbert, O., et al. 2012, A\&A, 539, A31 Daddi, E., Cimatti, A., Pozzetti, L., et al. 2000, A\&A, 361, 535 Daddi, E., Cimatti, A., Renzini, A., et al. 2004, ApJ, 617, 746 Davis, M., \& Geller, M. J. 1976, ApJ, 208, 13

Davis, M., Faber, S. M., Newman, J., et al. 2003, in SPIE Conf. 4834, ed. P. Guhathakurta, 161

Davis, M., Guhathakurta, P., Konidaris, N. P., et al. 2007, ApJ, 660, L1 de la Torre, S., Le Fèvre, O., Porciani, C., et al. 2011, MNRAS, 412, 825 Dickinson, M., Papovich, C., Ferguson, H. C., \& Budavári, T. 2003, ApJ, 587, 25

Fanidakis, N., Baugh, C. M., Benson, A. J., et al. 2011, MNRAS, 410, 53 Foucaud, S., Almaini, O., Smail, I., et al. 2007, MNRAS, 376, L20 Foucaud, S., Conselice, C. J., Hartley, W. G., et al. 2010, MNRAS, 406, 147 Gilbank, D. G., Bower, R. G., Glazebrook, K., et al. 2011, MNRAS, 414, 304 Gobat, R., Daddi, E., Onodera, M., et al. 2011, A\&A, 526, A133

Gonzalez-Perez, V., Baugh, C. M., Lacey, C. G., \& Almeida, C. 2009, MNRAS, 398, 497

Gonzalez-Perez, V., Baugh, C. M., Lacey, C. G., \& Kim, J. 2011, MNRAS, in press

Goranova, Y., Hudelot, P., Magnard, F., et al. 2009, The CFHTLS T0006 Release, http://terapix.iap.fr/cplt/T0006-doc.pdf Grazian, A., Fontana, A., Moscardini, L., et al. 2006, A\&A, 453, 507 Groth, E. J., \& Peebles, P. J. E. 1977, ApJ, 217, 385

Guzzo, L., Cassata, P., Finoguenov, A., et al. 2007, ApJS, 172, 254

Hamana, T., Ouchi, M., Shimasaku, K., Kayo, I., \& Suto, Y. 2004, MNRAS, 347, 813

Hartley, W. G., Lane, K. P., Almaini, O., et al. 2008, MNRAS, 391, 1301 Hartley, W. G., Almaini, O., Cirasuolo, M., et al. 2010, MNRAS, 407, 1212 Hartley, W. G., Almaini, O., Mortlock, A., et al. 2013, MNRAS, 431, 3045

Hasegawa, T., \& Umemura, M. 1993, MNRAS, 263, 191

Hayashi, M., Shimasaku, K., Motohara, K., et al. 2007, ApJ, 660, 72

Hilton, M., Lloyd-Davies, E., Stanford, S. A., et al. 2010, ApJ, 718, 133

Ilbert, O., Arnouts, S., McCracken, H. J., et al. 2006, A\&A, 457, 841

Ilbert, O., Salvato, M., Le Floc'h, E., et al. 2010, ApJ, 709, 644

Iovino, A., Giovanelli, R., Haynes, M., Chincarini, G., \& Guzzo, L. 1993, MNRAS, 265, 21

Kim, H., Baugh, C. M., Cole, S., Frenk, C. S., \& Benson, A. J. 2009, MNRAS, 400,1527

Kim, J., Edge, A. C., Wake, D. A., \& Stott, J. P. 2011, MNRAS, 410, 241

Kong, X., Daddi, E., Arimoto, N., et al. 2006, ApJ, 638, 72

Lagos, C. D. P., Lacey, C. G., Baugh, C. M., Bower, R. G., \& Benson, A. J. 2011, MNRAS, 416, 1566

Le Fèvre, O., Guzzo, L., Meneux, B., et al. 2005a, A\&A, 439, 877

Le Fèvre, O., Vettolani, G., Garilli, B., et al. 2005b, A\&A, 439, 845
Le Fèvre, O., Cassata, P., Cucciati, O., et al. 2013, A\&A, 559, A14 Le Fèvre, O., Tasca, L. A. M., Cassata, P., et al. 2014, A\&A, submitted [arXiv: 1403.3938]

Lewis, A., Challinor, A., \& Lasenby, A. 2000, ApJ, 538, 473

Li, C., Kauffmann, G., Jing, Y. P., et al. 2006, MNRAS, 368, 21

Lilly, S. J., Le Brun, V., Maier, C., et al. 2009, ApJS, 184, 218

Loveday, J., Maddox, S. J., Efstathiou, G., \& Peterson, B. A. 1995, ApJ, 442, 457

McCracken, H. J., Peacock, J. A., Guzzo, L., et al. 2007, ApJS, 172, 314

McCracken, H. J., Capak, P., Salvato, M., et al. 2010, ApJ, 708, 202

Meneux, B., Le Fèvre, O., Guzzo, L., et al. 2006, A\&A, 452, 387

Meneux, B., Guzzo, L., Garilli, B., et al. 2008, A\&A, 478, 299

Meneux, B., Guzzo, L., de la Torre, S., et al. 2009, A\&A, 505, 463

Mo, H. J., \& White, S. D. M. 1996, MNRAS, 282, 347

Mo, H. J., \& White, S. D. M. 2002, MNRAS, 336, 112

Moster, B. P., Somerville, R. S., Newman, J. A., \& Rix, H.-W. 2011, ApJ, 731, 113

Navarro, J. F., Frenk, C. S., \& White, S. D. M. 1997, ApJ, 490, 493

Nikoloudakis, N., Shanks, T., \& Sawangwit, U. 2013, MNRAS, 429, 2032

Noeske, K. G., Weiner, B. J., Faber, S. M., et al. 2007, ApJ, 660, L43

Norberg, P., Baugh, C. M., Hawkins, E., et al. 2001, MNRAS, 328, 64

Norberg, P., Baugh, C. M., Hawkins, E., et al. 2002, MNRAS, 332, 827

Onodera, M., Daddi, E., Gobat, R., et al. 2010, ApJ, 715, L6

Padilla, N. D., Christlein, D., Gawiser, E., et al. 2010, MNRAS, 409, 184

Peebles, P. J. E. 1980, The Large-Scale Structure of the Universe (Princeton University Press)

Phillipps, S., \& Shanks, T. 1987, MNRAS, 229, 621

Phillipps, S., Fong, R., Fall, R. S. E. S. M., \& MacGillivray, H. T. 1978, MNRAS, 182,673

Phillipps, S., Fong, R., \& Shanks, T. 1981, MNRAS, 194, 49

Pollo, A., Guzzo, L., Le Fèvre, O., et al. 2006, A\&A, 451, 409

Pozzetti, L., Bolzonella, M., Lamareille, F., et al. 2007, A\&A, 474, 443

Puget, P., Stadler, E., Doyon, R., et al. 2004, Ground-based Instrumentation for Astronomy, eds. A. F. M. Moorwood, \& I. Masanori, Proc. SPIE, 5492, 978

Quadri, R. F., Williams, R. J., Lee, K., et al. 2008, ApJ, 685, L1

Renzini, A., \& Daddi, E. 2009 [arXiv:0906.4662]

Roche, N., Shanks, T., Metcalfe, N., \& Fong, R. 1993, MNRAS, 263, 360

Roche, N. D., Almaini, O., Dunlop, J., Ivison, R. J., \& Willott, C. J. 2002, MNRAS, 337, 1282

Sánchez, A. G., Crocce, M., Cabré, A., Baugh, C. M., \& Gaztañaga, E. 2009, MNRAS, 400, 1643

Sawangwit, U., Shanks, T., Abdalla, F. B., et al. 2011, MNRAS, 416, 3033

Seljak, U., \& Zaldarriaga, M. 1996, ApJ, 469, 437

Shapley, A. E., Steidel, C. C., Pettini, M., \& Adelberger, K. L. 2003, ApJ, 588, 65

Sheth, R. K., Mo, H. J., \& Tormen, G. 2001, MNRAS, 323, 1

Simon, P. 2007, A\&A, 473, 711

Skibba, R. A., Smith, M. S. M., Coil, A. L., et al. 2014, ApJ, 784, 128

Springel, V., White, S. D. M., Jenkins, A., et al. 2005, Nature, 435, 629

Stalin, C. S., Petitjean, P., Srianand, R., et al. 2010, MNRAS, 401, 294

Stott, J. P., Collins, C. A., Burke, C., Hamilton-Morris, V., \& Smith, G. P. 2011 , MNRAS, 414, 445

Tanaka, M., Finoguenov, A., \& Ueda, Y. 2010, ApJ, 716, L152

Tinker, J. L., Weinberg, D. H., Zheng, Z., \& Zehavi, I. 2005, ApJ, 631, 41

Tinker, J. L., Leauthaud, A., Bundy, K., et al. 2013, ApJ, 778, 93

Tran, K.-V. H., Papovich, C., Saintonge, A., et al. 2010, ApJ, 719, L126

Wake, D. A., Sheth, R. K., Nichol, R. C., et al. 2008, MNRAS, 387, 1045

Wake, D. A., Whitaker, K. E., Labbé, I., et al. 2011, ApJ, 728, 46

Williams, R. J., Quadri, R. F., Franx, M., van Dokkum, P., \& Labbé, I. 2009 ApJ, 691, 1879

Zaldarriaga, M., \& Seljak, U. 2000, ApJS, 129, 431

Zheng, Z., Berlind, A. A., Weinberg, D. H., et al. 2005, ApJ, 633, 791

Pages 15 to 16 are available in the electronic edition of the journal at http://www . aanda.org 
Table A.1. Numbers of galaxies used in each mass/redshift bin.

\begin{tabular}{|c|c|c|c|c|c|c|c|c|c|c|c|c|}
\hline \multirow[t]{2}{*}{$z$} & \multicolumn{3}{|c|}{$10^{8.6}<M\left[h^{-1} M_{\odot}\right] \leq 10^{9.6}$} & \multicolumn{3}{|c|}{$10^{9.6}<M\left[h^{-1} M_{\odot}\right] \leq 10^{10.6}$} & \multicolumn{3}{|c|}{$10^{10.6}<M\left[h^{-1} M_{\odot}\right] \leq 10^{11}$} & \multicolumn{3}{|c|}{$M\left[h^{-1} M_{\odot}\right]>10^{11}$} \\
\hline & Blue & Red & All & Blue & Red & All & Blue & Red & All & Blue & Red & All \\
\hline 0.50 & 11239 & - & 13611 & 2525 & 1606 & 4131 & 329 & 719 & 1048 & - & - & 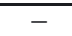 \\
\hline 0.70 & 23943 & - & 25133 & 8618 & 1849 & 10467 & 978 & 1142 & 2120 & - & 495 & 668 \\
\hline 0.90 & - & - & - & 7389 & - & 9790 & 1457 & 1266 & 2723 & 362 & 565 & 927 \\
\hline 1.10 & - & - & - & 9546 & - & 10444 & 1242 & 575 & 1817 & 254 & 217 & 471 \\
\hline 1.35 & - & - & - & 11180 & - & 12112 & 1795 & 655 & 2450 & 453 & 211 & 664 \\
\hline 1.75 & - & - & - & 32018 & - & 33677 & 2390 & - & 3406 & 706 & 454 & 1160 \\
\hline
\end{tabular}

\section{Appendix A: Galaxy numbers}

Table A.1 gives the numbers of galaxies per mass, redshift and galaxy type bin used in the clustering analysis. Bins not used in the analysis - due to either $<200$ galaxies or lying below our chosen mass limits at a given redshift - are denoted by "_".

\section{Appendix B: Excessive clustering in the COSMOS field at $z \sim 0.7$}

As noted in the main text, the D2/COSMOS field shows extremely elevated clustering levels compared to the three other fields in the redshift bin $0.6<z<0.8$. This is likely related to an over-density of galaxy clusters within this redshift bin, which are reported by a number of authors (e.g. Guzzo et al. 2007; Brusa et al. 2010). In our analysis, we have removed the COSMOS field from our calculations for the $z=0.7$ bins given that it significantly biases our results. For completeness, we show in Fig. B.1 the angular clustering measurements for the combination of the D1, D3 and D4 fields versus the measurement in the D2 field alone. The filled orange circles show the D2 result, whilst the black $\times$ symbols show the combined $\mathrm{D} 1+\mathrm{D} 2+\mathrm{D} 3$ result. In addition the green shaded region shows the maximum and minimum extent of the D1, D3 and D4 angular correlation functions. Clearly, at separations of $\theta \gtrsim 0.02^{\circ}$, the D2 result is an extreme outlier from the other three fields. At smaller separations the D2 measurement is in complete agreement with the other three fields. This fits with a simple model of there being a significant over-abundance of clusters in the D2 field, whereby the high number of clusters elevates the 2-halo term (large scales), whilst the clustering of galaxies within clusters themselves is unaffected and so the 1-halo term (small scales) remains the same.

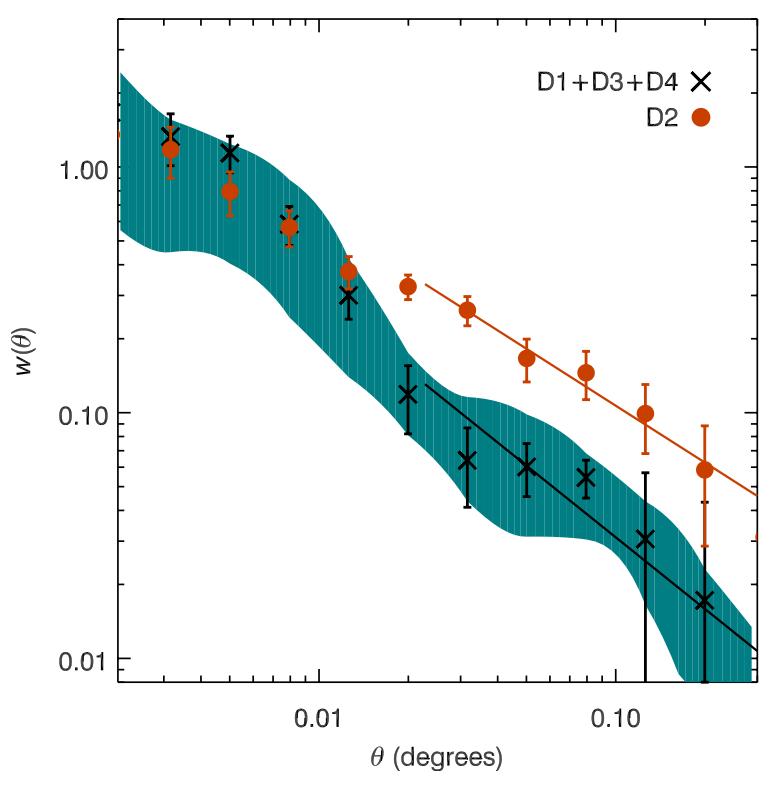

Fig. B.1. Comparison of the angular correlation function for $10^{10.6} h^{-1} M_{\odot}<M_{\star}<10^{11.0} h^{-1} M_{\odot}$ between the D2 field (orange circles) and the other three fields combined (black $\times$ ) at $0.6<z<0.8$. The shaded green region shows the maximum and minimum measurements taking the D1, D3 and D4 fields individually.

\section{Appendix C: Results of the $w(\theta)$ fitting}

We give the $A_{w^{-}} \gamma$ fits obtained for the fitting of the angular correlation function results for all considered combinations of galaxy mass, redshift and type in Table C.1. The corresponding values of the clustering length, $r_{0}$ (derived using the already determined values of $\gamma$ ), are provided in Table C.2. 
Table C.1. Fit parameters, $A_{w}$ (in units of $\operatorname{arcmin}{ }^{1-\gamma}$ ) and $\gamma$, for the correlation functions of all galaxies as a function of mass and redshift.

\begin{tabular}{|c|c|c|c|c|c|c|c|c|}
\hline \multirow[t]{2}{*}{$z$} & \multicolumn{2}{|c|}{$10^{8.6}<M\left[h^{-1} M_{\odot}\right] \leq 10^{9.6}$} & \multicolumn{2}{|c|}{$10^{9.6}<M\left[h^{-1} M_{\odot}\right] \leq 10^{10.6}$} & \multicolumn{2}{|c|}{$10^{10.6}<M\left[h^{-1} M_{\odot}\right] \leq 10^{11}$} & \multicolumn{2}{|c|}{$M\left[h^{-1} M_{\odot}\right]>10^{11}$} \\
\hline & $A_{w}$ & $\gamma$ & $A_{w}$ & $\gamma$ & $A_{w}$ & $\gamma$ & $A_{w}$ & $\gamma$ \\
\hline \multicolumn{9}{|c|}{ Passive+Star-forming } \\
\hline 0.50 & $0.12 \pm 0.02$ & $1.90 \pm 0.08$ & $0.17 \pm 0.04$ & $1.80 \pm 0.10$ & $0.27 \pm 0.07$ & $1.85 \pm 0.16$ & - & - \\
\hline 0.70 & $0.09 \pm 0.01$ & $1.85 \pm 0.03$ & $0.11 \pm 0.01$ & $1.83 \pm 0.06$ & $0.25 \pm 0.07$ & $1.76 \pm 0.10$ & $0.23 \pm 0.03$ & $1.63 \pm 0.09$ \\
\hline 0.90 & - & - & $0.11 \pm 0.02$ & $1.80 \pm 0.08$ & $0.25 \pm 0.09$ & $1.90 \pm 0.09$ & $0.24 \pm 0.07$ & $1.83 \pm 0.20$ \\
\hline 1.10 & - & - & $0.12 \pm 0.02$ & $1.90 \pm 0.04$ & $0.13 \pm 0.01$ & $1.62 \pm 0.18$ & $0.28 \pm 0.06$ & $1.72 \pm 0.27$ \\
\hline 1.35 & - & - & $0.07 \pm 0.02$ & $1.68 \pm 0.06$ & $0.14 \pm 0.03$ & $1.91 \pm 0.19$ & $0.44 \pm 0.07$ & $1.82 \pm 0.19$ \\
\hline 1.75 & - & - & - & - & - & - & $0.16 \pm 0.04$ & $1.94 \pm 0.21$ \\
\hline \multicolumn{9}{|c|}{ Passive } \\
\hline 0.50 & - & - & $0.34 \pm 0.11$ & $2.00 \pm 0.17$ & $0.53 \pm 0.23$ & $2.11 \pm 0.18$ & - & - \\
\hline 0.70 & - & - & $0.35 \pm 0.11$ & $1.82 \pm 0.07$ & $0.33 \pm 0.09$ & $2.00 \pm 0.11$ & $0.86 \pm 0.33$ & $2.05 \pm 0.19$ \\
\hline 0.90 & - & - & - & - & $0.56 \pm 0.18$ & $2.17 \pm 0.13$ & $0.44 \pm 0.30$ & $1.96 \pm 0.24$ \\
\hline 1.10 & - & - & - & - & $0.21 \pm 0.06$ & $1.86 \pm 0.15$ & $0.39 \pm 0.19$ & $1.94 \pm 0.20$ \\
\hline 1.35 & - & - & - & - & - & - & $0.76 \pm 0.43$ & $2.03 \pm 0.34$ \\
\hline 1.75 & - & - & - & - & - & - & $0.39 \pm 0.22$ & $2.10 \pm 0.13$ \\
\hline \multicolumn{9}{|c|}{ Star-forming } \\
\hline 0.50 & $0.09 \pm 0.02$ & $1.80 \pm 0.07$ & $0.17 \pm 0.05$ & $1.80 \pm 0.09$ & $0.19 \pm 0.10$ & $1.80 \pm 0.41$ & - & - \\
\hline 0.70 & $0.07 \pm 0.01$ & $1.71 \pm 0.05$ & $0.09 \pm 0.02$ & $1.86 \pm 0.06$ & $0.30 \pm 0.13$ & $1.89 \pm 0.15$ & - & - \\
\hline 0.90 & - & - & $0.11 \pm 0.01$ & $1.86 \pm 0.09$ & $0.18 \pm 0.05$ & $1.99 \pm 0.19$ & $0.54 \pm 0.20$ & $1.99 \pm 0.09$ \\
\hline 1.10 & - & - & $0.12 \pm 0.02$ & $1.89 \pm 0.04$ & $0.20 \pm 0.05$ & $1.90 \pm 0.15$ & $0.39 \pm 0.19$ & $1.77 \pm 0.17$ \\
\hline 1.35 & - & - & $0.07 \pm 0.00$ & $1.70 \pm 0.05$ & $0.11 \pm 0.04$ & $1.80 \pm 0.18$ & $0.32 \pm 0.14$ & $1.85 \pm 0.15$ \\
\hline 1.75 & - & - & - & - & - & - & $0.16 \pm 0.03$ & $1.85 \pm 0.17$ \\
\hline
\end{tabular}

Table C.2. Clustering lengths, $r_{0}$ (comoving and in units of $h^{-1} \mathrm{Mpc}$ ), for the correlation functions of all galaxies as a function of mass and redshift.

\begin{tabular}{|c|c|c|c|c|}
\hline$z$ & $10^{8.6}<M\left[h^{-1} M_{\odot}\right] \leq 10^{9.6}$ & $10^{9.6}<M\left[h^{-1} M_{\odot}\right] \leq 10^{10.6}$ & $10^{10.6}<M\left[h^{-1} M_{\odot}\right] \leq 10^{11}$ & $M\left[h^{-1} M_{\odot}\right]>10^{11}$ \\
\hline \multicolumn{5}{|c|}{ Passive+Star-forming } \\
\hline 0.50 & $3.3 \pm 0.3$ & $4.5 \pm 0.6$ & $5.5 \pm 0.8$ & - \\
\hline 0.70 & $3.2 \pm 0.2$ & $3.5 \pm 0.2$ & $5.7 \pm 0.8$ & $7.1 \pm 0.7$ \\
\hline 0.90 & - & $3.9 \pm 0.4$ & $5.2 \pm 1.0$ & $5.5 \pm 0.9$ \\
\hline 1.10 & - & $3.9 \pm 0.3$ & $4.6 \pm 0.2$ & $6.6 \pm 0.8$ \\
\hline 1.35 & - & $3.9 \pm 0.5$ & $4.8 \pm 0.5$ & $8.0 \pm 0.7$ \\
\hline 1.75 & - & - & - & $5.8 \pm 0.7$ \\
\hline \multicolumn{5}{|c|}{ Passive } \\
\hline 0.50 & - & $5.3 \pm 0.9$ & $5.4 \pm 1.1$ & - \\
\hline 0.70 & - & $7.2 \pm 1.3$ & $5.6 \pm 0.7$ & $7.7 \pm 1.4$ \\
\hline 0.90 & - & - & $5.5 \pm 0.8$ & $6.1 \pm 2.2$ \\
\hline 1.10 & - & - & $5.6 \pm 0.8$ & $6.5 \pm 1.6$ \\
\hline 1.35 & - & - & - & $8.5 \pm 2.4$ \\
\hline 1.75 & - & - & - & $7.2 \pm 1.9$ \\
\hline \multicolumn{5}{|c|}{ Star-forming } \\
\hline 0.50 & $3.0 \pm 0.4$ & $4.2 \pm 0.7$ & $4.4 \pm 1.3$ & - \\
\hline 0.70 & $3.1 \pm 0.2$ & $3.1 \pm 0.3$ & $5.5 \pm 1.2$ & - \\
\hline 0.90 & - & $3.9 \pm 0.3$ & $4.2 \pm 0.6$ & $6.8 \pm 1.2$ \\
\hline 1.10 & - & $3.8 \pm 0.3$ & $4.8 \pm 0.7$ & $4.5 \pm 1.2$ \\
\hline 1.35 & - & $3.9 \pm 0.0$ & $4.0 \pm 0.9$ & $6.7 \pm 1.5$ \\
\hline 1.75 & - & - & - & $6.9 \pm 0.7$ \\
\hline
\end{tabular}

Notes. The quoted figures use the corresponding values of $\gamma$ as given in Table C.1. 